\title{
Fundamental Limitations and State-of-the-art Solutions for Target Node Localization in WSNs
}

This paper was downloaded from TechRxiv (https://www.techrxiv.org).

LICENSE

CC BY 4.0

SUBMISSION DATE / POSTED DATE

$29-09-2021 / 11-10-2021$

CITATION

Caceres Najarro, Lismer Andres; Song, lickho; Kim, Kiseon (2021): Fundamental Limitations and State-of-theart Solutions for Target Node Localization in WSNs. TechRxiv. Preprint. https://doi.org/10.36227/techrxiv.16698469.v1

$\mathrm{DOI}$

10.36227/techrxiv.16698469.v1 


\title{
Fundamental Limitations and State-of-the-art Solutions for Target Node Localization in WSNs
}

\author{
Lismer Andres Caceres Najarro, Iickho Song, Fellow, IEEE, and Kiseon Kim, Senior Member, IEEE
}

\begin{abstract}
With the advances in new technological trends and the reduction in prices of sensor nodes, wireless sensor networks (WSNs) and their applications are proliferating in several areas of our society such as healthcare, industry, farming, and housing. Accordingly, in recent years attention on localization has increased significantly since it is one of the main facets in any WSN. In a nutshell, localization is the process in which the position of any sensor node is retrieved by exploiting measurements from and between sensor nodes. Several techniques of localization have been proposed in the literature with different localization accuracy, complexity, and hence different applicability. The localization accuracy is limited by fundamental limitations, theoretical and practical, that restrict the localization accuracy regardless of the technique employed in the localization process. In this paper, we pay special attention to such fundamental limitations from the theoretical and practical points of view and provide a comprehensive review of the state-of-the-art solutions that deal with such limitations. Additionally, discussion on the theoretical and practical limitations together with their recent solutions, remaining challenges, and perspectives are presented.
\end{abstract}

Index Terms-Cooperation, localization, practical limitations, signal of opportunity, theoretical limits, state-of-the-art, survey, virtual nodes, wireless sensor networks.

\section{INTRODUCTION}

$\mathbf{T}$ he continued evolution of wireless technology inevitably contributes to the proliferation of more wireless sensor networks (WSNs), which consist of a few, tens, hundreds, and even thousands of sensor nodes [1]. Numerous WSN applications will be interconnected with the immediate reach of the users or managers. Such interconnection may not need the intervention of humans. In fact, direct machine to machine communication is expected to prevail in future applications [2]. Fig. 1] shows some representative WSN applications that are starting to consolidate in our society. Smart cities are envisioned to optimize the efficiency of the cities for the benefit of people. For that purpose, several thousands of sensors may be required for gathering a variety of information related to energy, water, and transportation, for example [3]. Similarly, sensors are being used for the control, logistic, and management of the productivity of smart farms [4-6]. For instance, sensors can be placed underground for measuring

L. A. Caceres Najarro and K. Kim are with the School of Electrical Engineering and Computer Science, Gwangju Institute of Science and Technology, Gwangju 61005 Republic of Korea. Email: \{andrescn, kskim\}@gist.ac.kr.

I. Song is with the School of Electrical Engineering, Korea Advanced Institute of Science and Technology, Daejeon 34141 Republic of Korea, and also with Liangjiang International College, Chongqing University of Technology, Chongqing 401135 China. Email: i.song@ieee.org

This work was supported by the the National Research Foundation of Korea under Grant NRF-2021R1I1A1A01041257, and in part by the project titled "Development of Automatic Identification Monitoring System for Fishing Gears", funded by the Ministry of Oceans and Fisheries, Korea, for which the authors wish to express their appreciation.

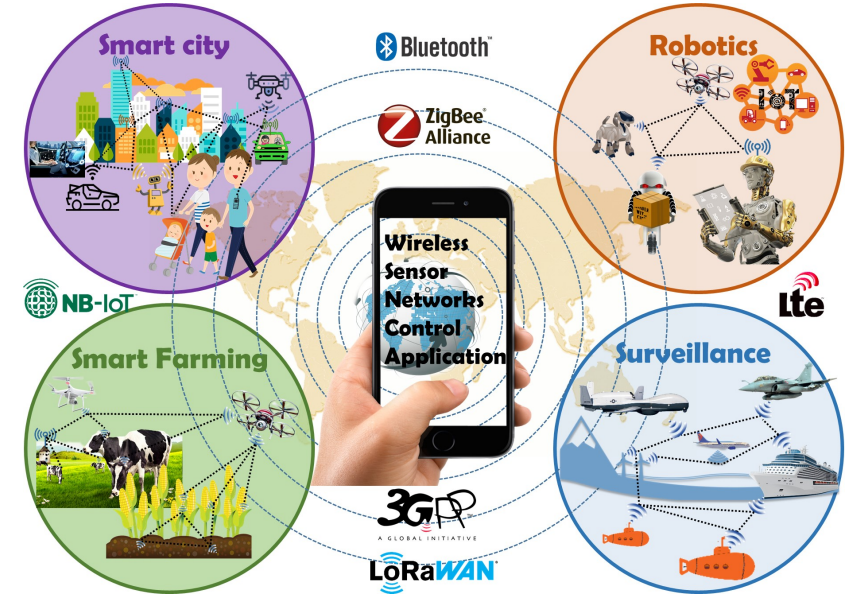

Fig. 1. Four representative applications of WSNs that are continuously developing.

the $\mathrm{pH}$, temperature, and humidity to enhance the productivity of crops; or above ground for measuring the direction and speed of animals for the purpose of monitoring. Surveillance is another application in which WSNs play an important role in the detection and tracking of airplanes, drones, and submarines [7]. Boosted by artificial intelligence and machine learning (ML) techniques [8]-10], robotics-related applications are continuously growing and will inevitably be the norm in the near future [11, 12].

The sensor nodes in WSNs are commonly grouped into two categories: anchor nodes (ANs) and target nodes (TNs), which are sensor nodes with known and unknown positions, respectively. To find the unknown locations of the TNs, some particular information related to the transmitted and/or received signal together with the position of the ANs are required. The information can be obtained from time, bearing, power, and, more recently, channel related measurements. Some examples include the time of arrival (TOA) [13, 14], angle of arrival (AOA) [15, 16], received signal strength (RSS) [17, 18], and channel state information (CSI) [19, 20]. These signal related measurements can be employed independently or combined with different levels of accuracy [21, 22]. Several techniques based on such measurements have been developed throughout recent years with their own pros and cons [2330]. However, regardless of the technique employed in the localization process, there exist theoretical fundamental limits that bounds theoretically the accuracy of any localization approach. Additionally, there are also practical fundamental limitations that prevent any localization technique to achieve the maximum reachable localization accuracy. 
Due to the rapid advancement in localization techniques, technologies, systems, and applications in WSNs, several review papers related to localization have been published in recent years. Although there exist many localization-related survey papers, most of them do not discuss or just slightly mention fundamental theoretical limits and practical limitations in the localization on WSNs. For instance, [23] surveyed enabling technology for localization, tracking, and navigation purposes. In [24], emphasis was put on the localization based on $5 \mathrm{G}$ technology where industrial, commercial, and academic solutions were summarized. Similarly, with a focus on cooperative localization schemes, a survey on algorithms and practical issues in applications with $5 \mathrm{G}$ and internetof-things technology was presented in [25]. Recently, [26] provided a review on common systems and technologies for indoor localization, and emphasized localization techniques for localizing human users and their devices. Likewise, [27] surveyed test and evaluation methodologies for localization systems focusing on pedestrian localization where the shortcomings of the current methodologies were highlighted. There also exist review papers that focus their attention on the applicability of localization techniques. For instance, [28] compared several localization techniques and analyzed their potential applicability to autonomous vehicle applications. In [29] the main wireless systems were surveyed in terms of techniques, principles, and structure for room monitoring, indoor localization, and activity recognition for health care applications. More recently, a comprehensive review focusing on the localization systems, techniques, errors and mitigation, and applications was provided in [30].

Unlike most of the review papers mentioned above, we focus in this paper our attention more on the theoretical fundamental limits where, besides the well known CramerRao lower bound (CRLB), tighter lower bounds are presented, grouped, and analyzed in detail. Additionally, further attention is given to the localization limitations found in practice, where such limitations are divided into general and particular limitations. General limitations refer to the limitations that restrict the localization accuracy regardless of the measurement employed in the localization process such as the availability of reference nodes, placement (geometry) of sensor nodes, multipath (MP) propagation, and non-line of sight (NLOS) conditions. Particular limitations refer to the limitations that only affect the localization accuracy according to the measurements employed in the localization process, e.g., synchronization in TOA based localization. For each of the practical limitations, a comprehensive review of the state-of-the-art solutions is presented and discussed with more attention to their limitations and possible improvement options.

The remainder of this paper is organized as follows. In Section II] fundamental theoretical limits are introduced in the form of bounds, where a general taxonomy of such fundamental bounds is presented. The pros and cons of each of the bounds are also described. In Section III practical fundamental limitations which are divided into general and particular limitations are explained. Section IV presents the state-of-the-art solutions for the general limitations presented in Section III Section V discusses perspectives and remaining challenges when dealing with fundamental practical limitations introduced in Section IV Finally, in Section VI concluding remarks are included.

\section{ThEORETICAL FUndAMENTAL Limits}

Let us consider a simplified measurement model for the localization problem: $y_{n}=s(\boldsymbol{\theta})+v_{n}$, for $n=1,2, \cdots, N$, where $y_{n}$ is the signal related measurement, which can be related for example to the RSS, TOA, or AOA; $v_{n}$ denotes the noise term with a probability density function (pdf); and $s(\cdot)$ denotes a function such that $s: \mathbb{R}^{D} \rightarrow \mathbb{R}$. Here, $\boldsymbol{\theta} \in \mathbb{R}^{D}$ represents the variable to be estimated, i.e., the unknown position of a TN in a $D$-dimensional space with $D=2$ or $D=3$. Note that $\boldsymbol{\theta}$ may also include other unknown model parameters increasing its dimension, e.g., path-loss exponent or transmit power in RSS based localization. Then, the localization problem consists in estimating the unknown position $\boldsymbol{\theta}$ of a TN by using the set $\boldsymbol{y}=\left\{y_{n}\right\}_{n=1}^{N}$ of measurements. Note that the relationship between $\boldsymbol{\theta}$ and $\boldsymbol{y}$ is described by the pdf $p(\boldsymbol{y} \mid \boldsymbol{\theta})$ of $\boldsymbol{y}$ parameterized by $\boldsymbol{\theta}$ and joint pdf $p(\boldsymbol{y}, \boldsymbol{\theta})$ when $\boldsymbol{\theta}$ is deterministic and random, respectively.

Although the localization problem in WSN has been studied extensively resulting in several interesting techniques, the localization accuracy of these techniques is bounded by theoretical limits. In other words, any localization technique cannot perform better than the maximum achievable accuracy dictated by theoretical bounds. The theoretical bounds help to understand how well a localization algorithm will perform in a certain configuration of sensor nodes. Additionally, it provides a baseline to compare several localization algorithms. One of the most popular bounds is the CRLB. However, it is known that this bound is just for unbiased localization estimators [31]. It has also been shown that the CRLB tends to be loose at low and medium signal-to-noise ratio (SNR) [32].

Apart from the CRLB, there exist various theoretical lower bounds that can be used for assessing properly the performance of localization algorithms. Fig. 2 shows a general taxonomy of the theoretical bounds. Based on the characteristic of the parameter to be estimated, theoretical lower bounds can be divided into three groups, namely, deterministic, random, and hybrid. These three groups can be further subdivided into two categories each based on the mathematical principle used: covariance inequality and detection formulation. The lower bounds in the group based on the covariance inequality use a special form of the Cauchy-Schwarz inequality [33]. In contrast, the lower bounds in the group based on detection formulation use detection strategies in accordance with the hypothesis testing formulation [31]. It is noteworthy that within each group, some bounds are generalizations of others. In the following subsection details of these bounds are provided.

\section{A. Families of Theoretical Lower Bounds}

\section{1) Cramer-Rao Lower Bound Family}

The CRLB provides the minimum limit of the variance of an estimator for deterministic parameters [34-36]. The CRLB limits the variance of any unbiased estimator by taking the inverse of the Fisher information (FI), which measures 


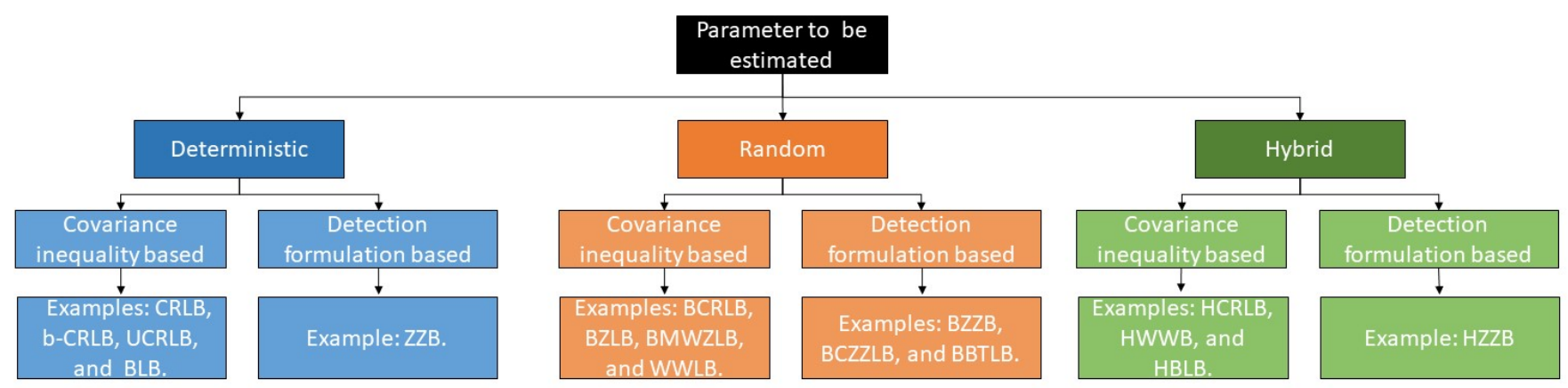

Fig. 2. General taxonomy of theoretical lower bounds based on the characteristic of the parameter to be estimated.

the amount of information contained in collected measurements. To reduce the variance of any estimator, independent measurements with relevant information should be taken into account [31]. Intuitively, increasing the FI results in a lower CRLB. The Cramer-Rao inequality holds only for unbiased estimators hence the CRLB does not provide a lower bound for biased estimators. For instance, some estimators based on RSS measurements have been demonstrated to be biased, and thus the mean square error (MSE) value will be lower than the CRLB in some regions [18, 37].

An extension of the CRLB in the case of biased estimator was introduced in [38], which states that the variance of any estimator with a given bias is bounded by the biased CRLB (b-CRLB). Interestingly, it turns out that the b-CRLB depends only on the bias gradient matrix, which implies that any constant bias can be neglected [38]. Taking into consideration the fact that a suitable bias gradient may reduce the b-CRLB, the uniform CRLB (UCRLB) was proposed [39]. The UCRLB exploits the bias-variance trade-off to provide a bound lower than the b-CRLB. Taking into account the same consideration, the UCRLB has been improved in several works [33, 40-42].

To partially avoid the limitation of the CRLB, prior information on the unknown position $\boldsymbol{\theta}$ of the TN can be included in the calculation of the CRLB, resulting in the Bayesian CRLB (BCRLB) [38]. In the BCRLB, instead of using the parameterized pdf $p(\boldsymbol{y} \mid \boldsymbol{\theta})$, the joint pdf $p(\boldsymbol{y}, \boldsymbol{\theta})$ is used to calculate the FI. Although the BCRLB can be used for unbiased and biased estimators, it requires two conditions for being used. First, the partial derivative of the joint pdf $p(\boldsymbol{y}, \boldsymbol{\theta})$ with respect to $\boldsymbol{\theta}$ should exist. Second, the FI matrix (FIM) needs to be invertible [43]. In consequence, in scenarios where mainly the NLOS prevails such as urban scenarios, use of the BCRLB may be inadequate. This is due to the fact that NLOS creates discontinuities that invalidate the first condition [44]. Additionally, it was argued that the prediction accuracy of the BCRLB deteriorates at low and medium SNR [32], which means that the BCRLB, like the CRLB, will serve as a good lower bound only at high SNR. It is worth mentioning that a tighter BCRLB can be found by taking into account the tradeoff between the variance and bias of an estimator [45, 46].

There also exists the hybrid CRLB (HCRLB), which provides a lower bound of the variance of an estimator in hybrid estimation problems [47]. In a hybrid estimation problem deterministic and random parameters are estimated simultaneously, i.e., $\boldsymbol{\theta}$ is composed of deterministic and random variables. For instance, consider the localization problem based on RSS measurements in which the estimation not only of the unknown position of the TN but also of the path-loss exponent is required. It has been demonstrated that the HCRLB is looser than the CRLB [48]. However, in many applications where nuisance parameters exist, the HCRLB may be more convenient than the CRLB due to the mathematical tractability [49].

\section{2) Barankin Lower Bound Family}

Similar to the CRLB, the Barankin lower bound (BLB) provides the lower bound on the variance of any unbiased estimator of deterministic parameters. It has been demonstrated that the BLB, in its more general form, provides the best possible lower bound on the variance of any unbiased estimator [50]. In contrast to the CRLB, the BLB does not require the existence of the partial derivative of the parameterized pdf $p(\boldsymbol{y} \mid \boldsymbol{\theta})$, and hence it can also be used for scenarios with the prevalence of NLOS conditions. It has been shown that the BLB is tight not only at high SNR but also at low and medium SNR [51-53].

The main drawback of the BLB is, however, the high mathematical complexity, generally without an analytical solution [54]. Although the BLB can be solved numerically, it requires to solve several optimization problems with respect to the testing points, admissible values of the parameter to be estimated. To deal with this drawback, several studies have been devoted to approximate the BLB [52, 54, 55]. The use of efficient algorithms may help reduce the calculation complexity [56].

The Bobrovsky-Zakai lower bound (BZLB) can be considered as an extension of the BLB on the estimation error for random parameters, which can be used also for assessing biased estimators. Interestingly, it was shown that the BCRLB is a special case of the BZLB [57]. Several works have demonstrated that the BZLB provides a tighter bound and predicts better the SNR threshold value [58, 59], which is important for setting the transmit power for adequate function of the localization system.

The hybrid BLB (HBLB) for providing a lower bound on the variance of hybrid parameters was introduced in [60]. Therein, it was shown that the CRLB, BCRLB, and HCRLB are special cases of the HBLB and that the HBLB is tighter than the 
HCRLB. However, a disadvantage of the HBLB is that it may not be the greatest lower bound on the estimation error for deterministic parameters [60]. An improved version of hybrid bounds has been recently reported [61] by combining the Barankin and Weiss-Weinstein lower bound (WWLB), which will be described shortly.

\section{3) Weiss-Weinstein Lower Bound Family}

The WWLB family is a specialized group of bounds for providing the lower bound on the estimation error of random parameters. The lower bounds in this family are calculated based on the covariance inequality principle for which the prior information related to the unknown random parameter is employed. This family includes the Bobrovsky-Mayer-WolfZakai lower bound (BMWZLB) [62], Bayesian Bhattacharyya lower bound [38], BCRLB [38], BZLB [57], and WWLB [63]. Among these bounds, the WWLB is the most popular since it was shown that several lower bounds such as the BCRLB, BZLB, and BMWZLB are special cases of the WWLB [64, 65]. Consequently, the general WWLB possesses the same advantages as its particular bounds, i.e, BCRLB, BZLB, and BMWZLB. In other words, the WWLB can also be used for unbiased and biased estimators and it also provides a tighter prediction at all SNR ranges, i.e., from low to high SNR, and even when the number of signal related measurements is small [66, 67]. In the worst case, the WWLB can provide similar predictions as the BCRLB. Up to date, the WWLB is considered the tightest lower bound for estimators of random parameters.

Although the WWLB provides a better lower bound in comparing localization algorithms, it usually requires an optimization procedure that in most cases may be highly computationally demanding. For some localization scenarios, it is possible to find a closed-form expression for the WWLB [68]. In other applications also a closed-form WWLB may exist [69].

The hybrid WWLB (HWWLB) combines the WWLB for the random parameter with other bounds for the deterministic parameter, such as the BLB and CRLB [61, 70]. In general, the hybrid bounds are not a simple concatenation of bounds for deterministic and random parameters, but a careful combination of them. A simple concatenation would not provide a proper lower bound when there exists dependence between the deterministic and random parameters.

\section{4) Ziv-Zakai Lower Bound Familiy}

Recently, based on the second order Taylor series approximation, an approximate Ziv-Zakai lower bound (ZZLB) for a deterministic parameter was derived for the TOA estimation problem [71]. An approximate ZZLB for deterministic parameters was also introduced [72]. Unfortunately, this approximation provides a lower bound on the estimation error only for unbiased estimators. However, it is worth mentioning that these ZZLB approximations provide a tighter bound not only at high SNR but also at medium and low SNR. It was shown that they also reasonably predict the threshold phenomenon.

Contrary to the ZZLB for deterministic parameters, the Bayesian ZZLB (BZZLB) was introduced several decades ago for a random parameter [73]. Later, its improved versions, known as the Bayesian Bellini-Tartara lower bound (BBTLB) [74] and Chazan-Zakai-Ziv lower bound (BCZZLB) [75] and the extended BZZLB for random parameters were introduced [76]. Using the nearest neighbor detection strategy, the ZZLB and BZZLB express the MSE in terms of hypothesis testing problem in which the probability of error corresponding to the nearest detector is replaced by an optimal detection strategy, e.g., maximum a posteriori. The BZZLB can be used for predicting the MSE or equivalently the root MSE for both biased and unbiased estimators, as the BCRLB does. The BZZLB provides a tighter prediction not only at high SNR but also at low SNR. The BZZLB also incorporates prior knowledge of the parameter. However, unlike the BCRLB, it does not depend on the distribution of the prior information [77]. A main drawback of the BZZLB is that it requires complex derivations that in most cases do not result in a closed-form solution. Nonetheless, a closed-form of the BZZLB can be found for some particular applications such as the estimation of the direction of arrival in radar systems [32, 78].

The hybrid ZZLB (HZZLB) was introduced in [79] by carefully combining the BLB and BZZLB for the deterministic and random parameters, respectively. It was shown that the HZZLB is as tight as the HWWLB at low, medium, and high SNR. The HZZLB performs better than the HCRLB and HBLB, especially at a medium SNR.

\section{B. Discussion}

In the case of estimating a deterministic parameter, there exist several bounds on the estimation error, for instance, the CRLB. Although the CRLB is by far the easiest to calculate, it provides an adequate lower bound only at a high SNR and when a large number of samples is available. In highly noisy scenarios where the number of samples is limited, the BLB and ZZLB are recommended since they provide tighter bounds at all the ranges of the SNR. However, it should be stressed that the CRLB, BLB, and ZZLB are limited to be used only for unbiased estimators. In the case of biased estimators, variants of the CRLB can be used, namely, the bCRLB and UCRLB.

In the case of estimating a random parameter, Bayesian bounds provide a lower bound indistinguishably for biased and unbiased estimators. Among the Bayesian bounds, the BCRLB is the most popular due to its mathematical tractability. Similar to the CRLB, the BCRLB provides a tight bound only at high SNR. Thus, other Bayesian bounds such as the BZLB, WWLB, and BZZLB are recommended when the localization system works at low SNR or constrained by few signal related measurements. Although there is no clear consensus on which Bayesian bound provides the best lower bound, the WWLB and BZZLB usually provide comparative performance and tighter bounds than others. A main drawback of the WWLB and BZZLB, however, is their lack of tractability that in most cases a closed-form solution may be difficult to find or even may not exist.

When dealing with the estimation of hybrid parameters, the HCRLB provides the same advantages and disadvantages as the CRLB and BCLRB. In consequence, the HZZLB and HWWLB are recommended since they have comparative 
performance and provide tighter bounds at low, medium, and high SNR. Here as well, the main drawback of the HZZLB and HWWLB is the lack of tractability. It is noted that approximation of the HZZLB and HWWLB in a closed-form may exist under some specific assumptions [79].

\section{Practical Fundamental Limitations}

The practical fundamental limitations are governed by several factors that constrain the localization accuracy of localization algorithms. These limitations can be broadly divided into two groups: general and particular limitations. General limitations denote a group of limitations that constrain the localization accuracy regardless of the signal related parameter used in the localization process. In contrast, particular limitations refer to a group of limitations that constrain the localization accuracy depending on the specific signal related measurements (e.g., time, angular, and strength) employed in the localization process.

\section{A. General Limitations}

\section{1) Availability of Reference Nodes}

The minimum number of ANs in two-dimensional (2D) and three-dimensional (3D) spaces is three and four, respectively, for localization techniques using signal strength and timerelated measurements. In contrast, if angle related measurements are used at least two and three ANs are required for 2D and 3D localization, respectively. Several works have shown that as the number of ANs increases the localization accuracy increases [18, 37, 80]. This result can be expected since more ANs result in more information related to the TN. However, there may be scenarios in which the number of available ANs is scarce for various reasons such as battery exhaustion, limited communication range, etc. In such scenarios the localization accuracy is limited.

\section{2) Placement of Sensor Nodes}

The placement of sensor nodes in the area of interest plays an important role in any localization algorithm regardless of the measurement used in the localization process. The placement of sensor nodes, specifically the placement of ANs, can be divided into two groups: homogeneous and nonhomogeneous. A placement of ANs is called nonhomogeneous when the ANs are placed only in some portion of the area of interest; otherwise, it is called homogeneous. The nonhomogenous placement of ANs does not assure that all the positions of the target nodes reside inside the convex hull formed by the ANs, which usually results in a lower localization accuracy than when the ANs are placed homogeneously.

An important parameter that helps interpret the effect of sensor nodes distribution on the localization accuracy is the geometric dilution of precision (GDOP). The localization accuracy of an algorithm increases as the value of GDOP decreases and the other way around [81]. The GDOP is a useful parameter in optimizing the deployment of sensor nodes.

\section{3) Multipath Propagation}

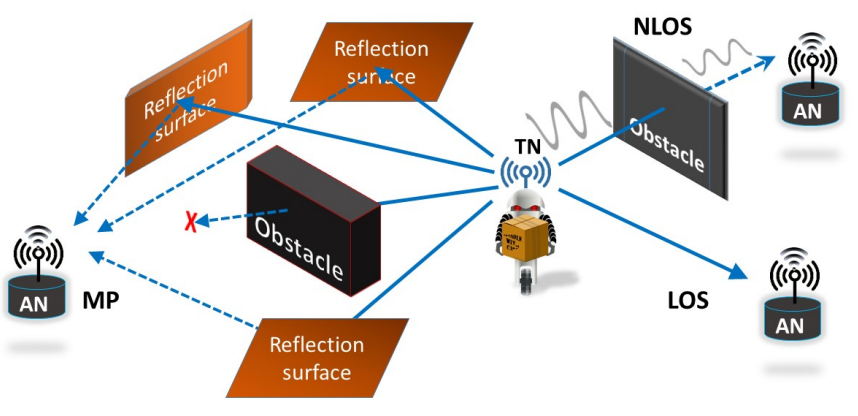

Fig. 3. A graphical representation of possible connection conditions between a TN and ANs: LOS, NLOS, and MP conditions. It is worth mentioning that in a connection between a TN and ANs, a condition may be present independently or in a combination of them.

The MP is a fundamental characteristic of radio propagation in WSNs. In a nutshell, MP propagation is a phenomenon in which the transmitted signal arrives at the destination receiver by two or more paths, as shown in Fig. 3 Once the transmitted signal arrives at the receiver, the MP causes constructive and destructive interference which conveys in phase shifting, changing (increasing or decreasing) the peak of the signal [82]. This situation alters the signal related measurements and hence the localization accuracy will be affected. For instance, in the RSS based localization, the destructive interference may lead to imply that the transmitter sensor is farther away than where the sensor node in fact is. Generally, MP propagation due to destructive and constructive interference will cause a negative or positive bias. In case the information of the propagation paths is determined, it can be used to improve the localization accuracy [83]. Nevertheless, getting this information requires more sophisticated sensor nodes with a higher number of antennas, which is usually prohibited in WSNs since the sensor nodes are small in size and generally constrained by computational power and energy.

\section{4) Non-line of Sight}

The NLOS is a phenomenon in which the LOS, also called the direct radio propagation path, between the transmitter and receivers is obstructed, thus degrading the quality of the radio signal, as shown in Fig. 3. In the worst case, it will cause the loss of the connection between the sensor nodes limiting the localization accuracy since the number of reference nodes will be reduced. The effects of NLOS will be stronger when the elements in the area of interest and surroundings are constantly changing (e.g., people moving). For time and signal strength related measurements, the NLOS causes positive bias. If there exist information on the NLOS links, it can be used to improve the localization accuracy.

\section{B. Particular Limitations}

The main particular limitations are divided into three signal related measurements, time, signal strength, and angle.

The synchronization among the sensor nodes is the main limitation regarding time related measurements. Generally, it is assumed that the sensor nodes have a common time basis. However, in practice, each sensor node in a WSN has its own 
oscillator (local clock) which undergoes unique degradation in time causing skew and offsets which will ultimately limit the localization accuracy [84]. This issue can be, to some extent, mitigated by considering round-trip or differential schemes [85, 86]. Another approach to mitigate synchronization issues is based on a virtual synchronization approach [87, 88].

Transmitted signals have particular characteristics that are used for localization. For instance, the strength of the transmitted signal decreases according to the distance between the transmitter and receiver. Ideally, it is considered that the strength measurement should be the same regardless of the receiver nodes when they are at the same distance from the transmitter node. However, this is far from reality. In fact, it has been proven that even if the receiver sensor nodes use the same chipsets, the strength measurements vary [89, 90]. These variations may be even bigger if the chipset vendors are different.

Another limitation related to the signal related measurements appears specifically when a parametric approach is used to estimate the location of a TN. A parametric approach models mathematically the signal propagation and its relation with the TN position [91]. Several works [37, 92,-94] use simplistic path loss model which may not correspond with the actual scenario. For instance, if the localization process is conducted over the sea surface, one-slope path loss model will not accurately represent the signal propagation. In fact, it was argued that more complex path loss models are required such as the two-ray or even the three-ray path loss model [95]. In other words, according to a certain scenario and variations, an appropriate path loss model should be employed in case it is desired to guarantee a good localization accuracy.

The main limitation when retrieving angular information for the localization process is the number of antenna elements included in a sensor node. To acquire the angular information from the transmitted signal arrived at the receiver, it is necessary to have two or more antenna elements. Increasing the number of antenna elements results in detecting more TNs and, at the same time, in the enhancement of the estimation accuracy of the angular information [96]. However, having more antennas requires paying extra attention to coupling and uneven gains effects [97]. To some extent, this situation can be mitigated with new approaches based on nesting techniques [98].

\section{RECEnt Solutions}

In this section, we introduce recent solutions for the general limitations introduced in Section III-A, i.e., availability of sensor nodes, placement of sensor nodes, MP propagation, and NLOS conditions. We focus our attention on the state-of-theart solutions for such limitations because they affect any localization technique regardless of the measurement employed in the localization process, contrary to the particular limitations. Recent solutions for the particular limitations related to time, signal strength, and angular based localization techniques can be found in [99-101], [17, 102], and [103-105], respectively.

\section{A. Increasing Availability of Reference Nodes}

A way to counteract the lack of ANs in the localization problem is to employ a cooperative localization scheme. Unlike a noncooperative scheme where only the ANs participate actively in the localization process, in the cooperative scheme both ANs and TNs participate actively taking measurements of the signal related parameters. Due to the active participation of the TNs, it is possible to increase the number of reference nodes since the TNs can be regarded as pseudo-ANs. A comprehensive theoretical and practical review of cooperative localization can be found in [119].

Another way to increase the number of reference nodes is by exploiting sensor nodes that are opportunistically in the proximity of the TNs, which is called opportunistic localization [106, 107]. Table $\mathbb{I}$ shows recent approaches for increasing the number of reference nodes based on opportunistic localization. The principle of opportunistic localization is similar to the cooperative scheme in the sense that it takes advantage of the sensor nodes that are near the TNs. The opportunistic localization is based on the idea that a $\mathrm{TN}$ interacts on an occasional basis only rather than in controlled or semi controlled way [106]. Additionally, in the opportunistic localization, the sensor nodes that enter in contact with the TN provide not only signal related measurements but usually their own location as well [107, 108].

There is a broader concept called the signal of opportunity (SOP) in which heterogeneous technologies are combined for extracting additional information such as time, power, angular, and movement patterns that helps overcome the limitation related to the number of ANs [116]. For instance, it was demonstrated that the time information can be retrieved from periodically transmitted signals that possess preambles such as those present in several technologies, e.g., cellular technology [109-111]. Recently, it was shown that by employing code division multiplexing access (CDMA) and long-term evolution (LTE) signals in a TN composed with a software-defined receiver, base stations of cellular networks can be exploited as additional reference nodes for increasing localization accuracy [110]. Therein, the employed ranges in the localization process were obtained by extracting time information from CDMA/LTE signals. Interestingly, it was demonstrated that the RSS, carrier phase, and direction of arrival can also be extracted from cellular signals, which may contribute further to the localization accuracy [111, 115, 117]. Additional improvement may be achieved by combining inertial measurement units (IMU) and vision data information [118]. The IMU measurements provide direction, angular rate, velocity, and relative position (given an initial position), which can be exploited for error mitigation [116, 120].

The exploitation of SOP can also be accomplished in underwater WSNs. A TN with limited ANs can exploit nodes external to its network (e.g., buoys and ships) as references for self localization [112-114]. The idea is to take advantage of the waveguide invariant property of the acoustic signal in underwater WSNs to extract the range between the TN and opportunistic nodes [113]. A further improvement can be achieved if the relative bearing information between the TN 
TABLE I

ReCENT APPROACHES FOR DEALING WITH LIMITED NUMBER OF ANCHOR NODES

\begin{tabular}{|c|c|c|c|c|}
\hline Ref. & Measurement & Technology & Solution & Remarks \\
\hline [106 & Not specified & Wi-Fi, Bluetooth & $\begin{array}{l}\text { Range-based localization where opportunistic refer- } \\
\text { ence nodes are scanned taking into account discovery } \\
\text { range, frequency, and duration. }\end{array}$ & $\begin{array}{l}\text { It is assumed that somehow the required } \\
\text { ranges are obtained by TOA or RSS. Energy } \\
\text { efficiency is not considered. }\end{array}$ \\
\hline 107 & Connectivity & $\begin{array}{l}\text { Zigbee, } \\
\text { Bluetooth }\end{array}$ & $\begin{array}{l}\text { Range-free-based localization in which floor plan is } \\
\text { exploited to constrain the movable areas of the TNs. } \\
\text { Reference nodes found opportunistically are included } \\
\text { in the multilateration process. }\end{array}$ & $\begin{array}{l}\text { Energy efficiency is not considered. Syn- } \\
\text { chronization among TNs is not required. } \\
\text { Low computational complexity and local- } \\
\text { ization accuracy. }\end{array}$ \\
\hline 108 & Time & Acoustic & $\begin{array}{l}\text { Interaction between TNs and potential reference } \\
\text { nodes is formulated under a game theory framework, } \\
\text { where the location of a TN is obtained by minimizing } \\
\text { the localization delay in a sparse WSN. }\end{array}$ & $\begin{array}{l}\text { Energy efficiency is considered. Synchro- } \\
\text { nization among the sensor nodes is required. } \\
\text { Computational time increases rapidly as the } \\
\text { number of sensor nodes increases. }\end{array}$ \\
\hline $109-111$ & Time, angular & CDMA, LTE & $\begin{array}{l}\text { Range/angle-based localization where periodicity of } \\
\text { cellular signal are exploited for retrieving ranges and } \\
\text { angular information between bases stations and the } \\
\text { TN. }\end{array}$ & $\begin{array}{l}\text { High complex hardware with limited num- } \\
\text { ber of processed signals. Energy efficiency } \\
\text { is not considered. }\end{array}$ \\
\hline $112-114$ & $\begin{array}{l}\text { Waveguide- } \\
\text { invariant } \\
\text { spectrum, } \\
\text { bearing }\end{array}$ & $\begin{array}{l}\text { Acoustic, Auto- } \\
\text { matic identifica- } \\
\text { tion system }\end{array}$ & $\begin{array}{l}\text { Range and bearing information between passing } \\
\text { ships and an underwater TN are exploited for self } \\
\text { localization. }\end{array}$ & $\begin{array}{l}\text { Energy efficiency is not considered. Initial } \\
\text { position of the } \mathrm{TN} \text { is required. }\end{array}$ \\
\hline 115 & Carrier phase & LTE & $\begin{array}{l}\text { Carrier phase observations are parameterized in } \\
\text { terms of the states, i.e., position and velocity, of the } \\
\text { TN and cellular base stations. Kalman filter is used } \\
\text { for tracking the position of the TN and unknown } \\
\text { parameters. }\end{array}$ & $\begin{array}{l}\text { The TN is required to be inside the convex } \\
\text { hull formed by the reference nodes for se- } \\
\text { curing good accuracy. Synchronization be- } \\
\text { tween cellular base stations is not required. }\end{array}$ \\
\hline 116 & $\begin{array}{l}\text { Signal power, an- } \\
\text { gular, time }\end{array}$ & $\begin{array}{l}\text { Acoustic, } \\
\text { Bluetooth, IMU }\end{array}$ & $\begin{array}{l}\text { Bluetooth and acoustic RSS measurements are em- } \\
\text { ployed for coarse localization. Then, the localization } \\
\text { is refined based on TOA of acoustic measurements } \\
\text { constrained to the movement pattern of the TN via } \\
\text { convex optimization. }\end{array}$ & $\begin{array}{l}\text { Initial position of the } \mathrm{TN} \text { is required. } \\
\text { High accuracy and computational complex- } \\
\text { ity. Reference nodes positions are known in } \\
\text { advance. Energy efficiency is not consid- } \\
\text { ered. }\end{array}$ \\
\hline 117 & Signal power & LTE & $\begin{array}{l}\text { The ordinary least square and Kalman filter ap- } \\
\text { proaches are employed for estimating the position of } \\
\text { a TN, which posses heterogeneous transmitters. Such } \\
\text { transmitters together with roadside ANs are exploited } \\
\text { to find the position of the TN. }\end{array}$ & $\begin{array}{l}\text { Low localization accuracy and computa- } \\
\text { tional complexity. Energy efficiency is not } \\
\text { considered. }\end{array}$ \\
\hline 118 & Signal power & LTE, IMU & $\begin{array}{l}\text { RSS measurements from heterogeneous reference } \\
\text { nodes are employed in a simple least square formu- } \\
\text { lation for preliminary estimation of the TN position. } \\
\text { Kalman filter supported with IMU measurements is } \\
\text { used for position refinement. Further refinement is } \\
\text { achieved via vision data. }\end{array}$ & $\begin{array}{l}\text { Initial positions of the TN and reference } \\
\text { nodes are not required. Low localization } \\
\text { accuracy and computational complexity. En- } \\
\text { ergy efficiency is not considered. }\end{array}$ \\
\hline
\end{tabular}

and opportunistic nodes is estimated [112]. It should be noted that apart from the SOP highlighted in Table I] there exist other SOPs that can also be exploited such as television and radio in their two formats, digital and analog [121].

\section{B. Optimized Placement of Reference Nodes}

The placement of ANs influences directly the localization accuracy regardless of the signal related parameters used in the localization process. Generally, the sensor nodes can be deployed randomly and deterministically. Although a random deployment may be convenient and in some cases unavoidable (e.g. inaccessible areas, military applications), random deployment of sensor nodes will generally provide lower localization accuracy. The lower localization accuracy is mainly due to 1) restricted communication coverage, which may result in insufficient reference nodes for the localization process; and 2) an inadequate TN-ANs geometrical configuration, which usually results in an ill-conditioned solution. A simple way to mitigate this situation is to increase the number of deployed ANs. However, from an economical point of view it may not be plausible since it will increase the deployment and maintenance cost. In contrast, deterministic deployment allows to optimize the placement of ANs not only for improving localization accuracy but also for improving communication coverage and even energy efficiency [130].

Table [II shows recent solutions for optimal deployment of ANs in terrestrial WSNs. A review of optimal deployments of ANs in underwater WSNs can be found in [131]. The optimal placement of the sensor nodes can be obtained by minimizing the GDOP. The GDOP is closely related to the CRLB [132], and consequently, the optimal placement can be obtained by maximizing the FIM. By reducing the value of GDOP, an improvement in the localization accuracy regardless of the algorithm employed for finding the position of the TNs can be secured [18, 133].

Several approaches employ directly or indirectly the GDOP as a metric for optimizing the placement of ANs. For instance, in [122] the optimal placement of ANs in an industrial scenario was considered by minimizing the average MSE of the position estimation based on TOA measurements, which was conducted based on the plane intersection method [134]. The optimal placement was given by finding the optimal separation between ANs, which interestingly was found in a closed-form. In [123] the placement of unmanned aerial vehicles (UAVs) 
TABLE II

Recent Approaches for Optimal Placement of Sensor Nodes

\begin{tabular}{|c|c|c|c|c|c|c|c|}
\hline Ref. & Scenario & $\begin{array}{l}\text { Deployment } \\
\text { type }\end{array}$ & Constraints & Measurements & Variables & Method & Remarks \\
\hline [123] & 2D Outdoor & Deterministic & $\begin{array}{l}\text { Equal distance be- } \\
\text { tween nodes (trian- } \\
\text { gular formation) }\end{array}$ & RSS & $\begin{array}{l}\text { Inter-node } \\
\text { distance, } \\
\text { altitude, } \\
\text { coverage } \\
\text { radius }\end{array}$ & $\begin{array}{l}\text { The localization MSE is } \\
\text { minimized with respect } \\
\text { to altitude constrained to } \\
\text { coverage radius. }\end{array}$ & $\begin{array}{l}\text { LOS and NLOS are con- } \\
\text { sidered. Known and equal } \\
\text { path-loss exponents are } \\
\text { assumed. }\end{array}$ \\
\hline 125 & 3D Outdoor & $\begin{array}{l}\text { Semi- } \\
\text { deterministic }\end{array}$ & $\begin{array}{l}m \text { randomly de- } \\
\text { ployed ANs and } 1 \\
\text { new AN position to } \\
\text { be optimized }\end{array}$ & TOA & $\begin{array}{l}\text { Inter-node } \\
\text { distance, } \\
\text { pseudorange }\end{array}$ & $\begin{array}{l}\text { The GDOP is minimized } \\
\text { by reformulating it as a } \\
\text { quadratic fractional pro- } \\
\text { gramming. }\end{array}$ & $\begin{array}{l}\text { Global optimal solution is } \\
\text { provided. NLOS is not } \\
\text { considered. }\end{array}$ \\
\hline 127] & 2D Outdoor & Deterministic & $\begin{array}{l}\text { TN outside of the } \\
\text { placement area of } \\
\text { ANs }\end{array}$ & $\begin{array}{l}\text { Not specified } \\
\text { (range-based) }\end{array}$ & $\begin{array}{l}\text { Inter-node } \\
\text { distance, } \\
\text { angle } \\
\text { between } \\
\text { TN and ANs }\end{array}$ & $\begin{array}{l}\text { The determinant of the } \\
\text { FIM is maximized con- } \\
\text { strained to the angles be- } \\
\text { tween TN and ANs. }\end{array}$ & $\begin{array}{l}\text { TN placed only outside } \\
\text { of the convex hull formed } \\
\text { by the ANs. NLOS is not } \\
\text { considered. }\end{array}$ \\
\hline [128] & 3D Indoor & Deterministic & $\begin{array}{l}\text { TN outside of the } \\
\text { placement area of } \\
\text { ANs }\end{array}$ & TOA & $\begin{array}{l}\text { Inter-node } \\
\text { distance }\end{array}$ & $\begin{array}{l}\text { The CRLB is minimized } \\
\text { based on GA considering } \\
\text { NLOS conditions. }\end{array}$ & $\begin{array}{l}\text { TN placed only outside of } \\
\text { the convex hull formed by } \\
\text { the ANs. NLOS is consid- } \\
\text { ered. }\end{array}$ \\
\hline [129] & 2D Outdoor & Deterministic & Not mentioned & $\begin{array}{l}\text { AOA, TOA, } \\
\text { RSS }\end{array}$ & $\begin{array}{l}\text { Inter-node } \\
\text { distance, } \\
\text { bearing }\end{array}$ & $\begin{array}{l}\text { The GDOP is minimized } \\
\text { under Bayesian } \\
\text { framework where the } \\
\text { uncertainty of the prior } \\
\text { knowledge of the position } \\
\text { of the TN is considered. }\end{array}$ & $\begin{array}{l}\text { Maximizing the determi- } \\
\text { nant of the FIM equivalent } \\
\text { to minimizing the modu- } \\
\text { lus of the sum of vectors } \\
\text { (TN, ANs). NLOS is not } \\
\text { considered. }\end{array}$ \\
\hline
\end{tabular}

as ANs for RSS-based localization was analyzed. Therein was demonstrated that there exists an optimal altitude and separation of the UAVs for improving the localization accuracy. It was also shown that the optimal altitude and separation of UAVs vary from urban to suburban scenarios. Recently, in [124] the optimal deployment of ANs was dealt in a scenario in which some ANs were already deployed and additional ANs were included for increasing the accruacy in the localization of a TN based on AOA measurements. Therein, it was demonstrated that, by reformulating the CRLB based on frame theory [135], a more tractable cost function for the optimal placement problem can be found. In [126], the frame theory was also employed for optimizing the placement of ANs when RSS measurements were considered. Interestingly, a scenario with random deployment of ANs was considered in [125], where the goal was to optimize the position of an additional AN for improving the overall localization accuracy based on TOA measurements. To achieve that goal, the weighted GDOP was minimized in a frame of quadratic fractional programming from which a global optimal solution was provided.

As it is mentioned above, there are scenarios in which the deployment of TNs and ANs are restricted to a certain area, e.g., deployments on the sea shores, mining areas, etc. In such scenarios, the optimal placement of ANs is also limited.
Taking into account such constraints, some works could still find the optimal placement of ANs. For instance, a restricted rectangular area where the ANs and TN can only be positioned inside and outside of the rectangular area, respectively, was considered in [128]. Therein, a genetic algorithm (GA) was employed for optimizing the placement of ANs. The fitness function of the GA was created based on the MSE of the localization problem. The lower the localization error, the better the fitness function of an individual. In [127] the optimal placement of ANs was dealt with in a more general framework in which the optimal placement of ANs was obtained based on finding optimal angles between the TN and ANs with no assumption on the geometry formed between them or the shape of the deployment area of the ANs, except for that a TN should be outside of the deployment area of the ANs.

Recently, the optimization of the placement of ANs was tackled in a unified manner considering AOA, TOA, RSS, and the combination of those measurements, where the uncertainty of the TN position was considered in a Bayesian framework [129]. It was shown that maximizing the determinant of the FIM is equivalent to minimizing the trace of the covariance matrix of the estimation error, and consequently the optimization of the placement of ANs could be formulated as the minimization of the modulus of a vector sum [129]. 
TABLE III

ReCEnt Approaches for DeAling With the Multipath Propagation

\begin{tabular}{|c|c|c|c|c|}
\hline Category & Type & Ref. & Solution & Limitation \\
\hline \multirow{3}{*}{ Mitigation } & Transceiver level & $136-141$ & $\begin{array}{l}\text { The mitigation is based on the polarization } \\
\text { difference between the DPC and IPCs. In- } \\
\text { creasing the number of antennas results in a } \\
\text { better mitigation of the effects of the IPCs. }\end{array}$ & $\begin{array}{l}\text { It increases the size and price of the sensor } \\
\text { nodes. }\end{array}$ \\
\hline & Medium access control level & 142,146 & $\begin{array}{l}\text { The FH technique is employed for mitigat- } \\
\text { ing the effects of the IPCs. The information } \\
\text { obtained in all the available channels is } \\
\text { used for increasing the ranging accuracy, } \\
\text { which will result in the improvement of the } \\
\text { localization accuracy. }\end{array}$ & $\begin{array}{l}\text { Create a high burden in the sensors' bat- } \\
\text { tery life due to high power consumption. } \\
\text { Ranging errors may increase due to hopping } \\
\text { impairments, which may disrupt the com- } \\
\text { munication between the TN and ANs. }\end{array}$ \\
\hline & Model level & 147,152 & $\begin{array}{l}\text { The effects of the MPCs are modeled as } \\
\text { additive biases. The sparsity of the MPCS } \\
\text { on the localization model can be exploited. }\end{array}$ & $\begin{array}{l}\text { The models tend to be non-convex and } \\
\text { highly nonlinear, which in most cases is } \\
\text { required to be relaxed. The relaxation may } \\
\text { limit the accuracy. }\end{array}$ \\
\hline \multirow[t]{2}{*}{ Exploitation } & Virtual nodes & 153.159 & $\begin{array}{l}\text { The IPCs are cast as DPCs between the } \\
\text { VNs and TN. The VNs are created by } \\
\text { mirroring the physical transmitter position } \\
\text { at the reflecting point. }\end{array}$ & $\begin{array}{l}\text { High computational complexity due to the } \\
\text { requirement of resolution and association of } \\
\text { the MPCs. Accuracy highly dependent on } \\
\text { the knowledge of TNs' environment and the } \\
\text { resolvability of the MPCs. }\end{array}$ \\
\hline & Machine learning & 160,163 & $\begin{array}{l}\text { Exploits the characteristics of the MPCs } \\
\text { such as the delay, phase, and amplitude } \\
\text { for creating a more reliable fingerprinting } \\
\text { database. }\end{array}$ & $\begin{array}{l}\text { It is time consuming to create a finger- } \\
\text { printing dataset and, in scenarios with high } \\
\text { variability, it may require to update the } \\
\text { fingerprinting data set from time to time. }\end{array}$ \\
\hline
\end{tabular}

\section{Mitigation and Exploitation of Multipath Components}

The MP components (MPCs), which are caused by the signal reflection, refraction, and scattering on the elements (e.g., walls, tables, people, etc.) surrounding the sensor nodes, can be grouped into direct path components (DPCs) and indirect path components (IPCs). The IPCs can further be grouped into two groups: specular and diffuse. The specular IPCs are the results of a perfect reflection of the transmitted signal. The diffuse IPCs are the results of scattering of the transmitted signal. In general, the MPCs introduce positive or negative bias to the localization process, which degrades the localization accuracy. There have been several efforts for mitigating and recently exploiting the MPCs for improving the localization accuracy, see Table III

\section{1) Mitigation of Multipath Components}

A common approach for mitigating the MPCs is to take advantage of the polarization difference between the DPCs and IPCs [164]. Recently, in [137] a single dual-polarized antenna has been proposed for the detection of the IPCs. Therein, a parallel cross-cancellation receiver is suggested for the mitigation of the IPCs. However, it was argued that the dual-polarization antennas may not perform always better than the one-polarization antennas for the IPCs mitigation. In that regard, [138] demonstrated that a dual polarization antenna provides better mitigation of IPCs than the single polarization antenna when there is a considerable difference between the received powers in the left and right circular polarized signals. More recently, [139] showed that by increasing the polarization diversity from two to four with linear and circular polarization antennas, it is possible to mitigate further the IPCs. Polarized antennas have been recently employed for mitigating the effects of MPCs in the localization of a TN with a single AN by employing Bluetooth technology [165].

Another approach to mitigate the MPCs is to employ
Bayesian and particle filters to track down parameters (e.g., amplitudes and delay) of the DPCs and IPCs. However, tracking the parameters of all the signals is highly computationally demanding. Recently, a particle filter with a reduced state signal model combined with a beam-forming technique was presented in [136]. Therein, the state received signal is modeled only in terms of the DPC while the IPCs are considered as part of the thermal noise, which are suppressed by employing a hybrid beamforming technique. Overall, the approach in [136] mitigates the MPCs with a reduced computational demand. More recently, beamforming was employed for reducing the effects of MPCs and increasing the localization accuracy by sequentially refining the beamforming vectors [140]. The mitigation of the IPCs can further be improved considering adaptive beam with width control [141]. Furthermore, it was theoretically demonstrated that, by increasing the number of antennas, the IPCs can be mitigated successfully and that the localization performance tends to coincide with that obtained when only the DPC is present [166].

Since the MPCs are strongly dependent on the carrier frequency and the surroundings of the sensor nodes, the effects of MPCs can also be mitigated by using several carrier frequencies in a controlled manner. Recently, a fast frequency hopping $(\mathrm{FH})$ technique has been employed not only for mitigating the effects of MPCs but also for reducing signal interference [142, 144]. It has also been shown that, by increasing the number of subcarriers, the mitigation of the IPCs can be enhanced [146]. Although FH techniques are useful for mitigating the effects of MPCs, there are open issues that need to be carefully addressed such as hop impairments (e.g., phased offset and group delay), especially for time-based localization techniques [145, 167]. In contrast, employing FH for mitigating the effects of MPCs in RSS-based localization techniques is much easier. It was demonstrated that by a simple averaging of the RSS measurements corresponding to each 
channel, mitigation of MPCs can be achieved [143, 168, 169]. Another option may be to solve a constrained multi objective function, which is constructed based on the channel diversity assumption [170].

The effects of MPCs can be modeled as a bias error parameter in the model of the localization problem, and can be solved by using several tools such as convex optimization and sparse estimation methods [150]. It was demonstrated that fuzzy logic can be a useful tool for inferring the MPCs error with considerably less computational demand [149]. Furthermore, when the sensor nodes change their positions in time, several techniques based on the extended Kalman filter and particle filter can be used to track the positions of the TN and bias error caused by the MPCs [148, 151, 171]. Although these techniques successfully mitigate the bias error caused by the MPCs, they tend to be highly computationally demanding due to an increased state vector. To avoid a large state vector, in [147] and [152] was exploited the sparsity of the bias error, under the assumption that only a few of the transmitters were affected by IPCs. In [147] the effects of MPCs are mitigated in a scenario using global navigation satellite system.

\section{2) Exploitation of Multipath Components}

For exploiting the MPCs, it is necessary to clearly distinguish between the DPC and IPCs. To do so, technologies that allow high resolvability in time and space are required.

Wide band (WB) and ultra WB (UWB) systems allow high resolvability of the MPCs in the time domain [172]. Consequently, instead of mitigating the MPCs, they can be exploited for increasing the localization accuracy. Once the MPCs are resolved, they can be seen as DPCs from virtual nodes (VNs), refer to Fig. 4. Based on such an idea, under the assumption of a static environment with a known $\mathrm{AN}$, known initial position and heading of the TN, an algorithm called Channelsimultaneous-localization-and-mapping was developed to find the position of the TN and VNs simultaneously [153]. The extension in the case of multiple TNs was recently proposed in [159], where it is shown that the localization accuracy is improved by increasing the number of TNs with the increase of MPCs. In contrast to [159] where cooperation between TN was not considered, [173] demonstrated that by increasing the number of cooperative nodes the localization accuracy increases even without the existence of ANs.

In bandwidth limited technologies such as the radio frequency identification (RFID) and wireless-fidelity (Wi-Fi), the resolvability of the MPCs in the time domain is limited. To overcome this limitation the use of multiple array antennas is an option since the array antennas provides resolvability of the MPCs in the angular domain [174]. Increasing the number of antennas will result in improving the resolvability of the MPCs and consequently the localization accuracy [154, 155]. Increasing the resolvability in the time and angular domain will enhance further the localization accuracy: millimeter-wave technology is expected to increase further the resolvability of MPCs in time and angular domain due to its wide bandwidth and multiple antenna arrays. Recently, this was theoretically demonstrated in [175] and verified through simulations in [156, 158] where the contributive information of the MPCs was analyzed.

Although the specular MPCs are the ones contributing more to the overall channel gain and exploited in the localization process, the diffuse MPCs can also be used for increasing the localization accuracy. Recently, it was demonstrated that the localization of a TN can be conducted even in the absence of the DPCs and specular IPCs [176, 177]. Furthermore, it was demonstrated that exploiting the diffuse IPCs improves not only the localization accuracy but also the synchronization [157].

The last decade has witnessed crucial breakthroughs in the field of ML and data science and the techniques developed therein are making their ways in the localization area [178]. The ML-based localization techniques are composed of two phases: offline and online. In the offline phase, the ML technique is trained by usually a large amount of data for mapping (learning) a relationship between the input data and desired output. In the online phase, the ML technique infers the output from new input data. The ML techniques depend on the quality and amount of data. In other words, as the quality and quantity of data grows, the learning becomes better. The characteristics of the MPCs, i.e, amplitude, delay, and phase, can be exploited for a better quality of data, which results in an improvement in the localization accuracy. Recently, it was demonstrated that, by employing the characteristics of MPCs in the fingerprinting dataset, the TDOA estimation could be considerably improved [161]. More recently, instead of using the CSI directly for the fingerprinting-based localization, the characteristics of the MPCs were extracted and employed for creating a refined fingerprinting database which provided an improved localization accuracy than its counterparts [162, 163]. The characteristic of the MPCs at different frequencies can also be used for increasing the quality of the fingerprinting dataset. For instance, by concatenating channel frequency responses at different frequencies, a better fingerprinting dataset can be obtained [160].

\section{Recent Solutions for Non-line of Sight}

In contrast to the effects of MPCs which can introduce positive or negative bias, the NLOS conditions only introduce positive bias. To deal with the bias error caused by the NLOS conditions, several works have been proposed in the literature, which can be grouped into two groups: identification and mitigation, see Table IV] In the identification group are the works that mainly focus on the LOS and NLOS classification. Once the LOS and NLOS conditions between the TN and ANs are determined, the ANs with NLOS condition can be discarded from the localization process. In contrast, in the mitigation group are the works that, instead of discarding the ANs with NLOS condition, they compensate the bias error caused by the NLOS condition. These works are useful especially in scenarios where the number of ANs is limited.

\section{1) Identification of Non-line of Sight}

The approaches employed for the identification of NLOS conditions can roughly be classified into two types: channelbased and range-based. The approaches in the channel-based 
TABLE IV

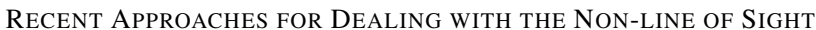

\begin{tabular}{|c|c|c|c|c|}
\hline Category & Type & Ref. & Solution & Limitations \\
\hline \multirow[t]{2}{*}{ Identification } & Channel-based & [179, 189 & $\begin{array}{l}\text { Mainly ML techniques that employ infor- } \\
\text { mation related to signal and/or channel } \\
\text { characteristics to distinguish the LOS and } \\
\text { NLOS. }\end{array}$ & $\begin{array}{l}\text { Specific characteristics of the LOS and } \\
\text { NLOS conditions are required. Performance } \\
\text { degradation in highly dynamic scenarios. } \\
\text { The performance and computational com- } \\
\text { plexity highly dependent on the size of the } \\
\text { training and testing data. }\end{array}$ \\
\hline & Range-based & 190, 194 & $\begin{array}{l}\text { Estimated ranges among the TN and ANs } \\
\text { and their characteristics in LOS and NLOS } \\
\text { are employed to distinguish NLOS condi- } \\
\text { tions }\end{array}$ & $\begin{array}{l}\text { The NLOS identification is highly depen- } \\
\text { dent on the detection threshold parameter, } \\
\text { which in most cases is considered fixed for } \\
\text { a specific scenario. }\end{array}$ \\
\hline \multirow{2}{*}{ Mitigation } & Direct & 195, 208 & $\begin{array}{l}\text { Does not require NLOS identification, } \\
\text { rather directly suppress the effect of NLOS } \\
\text { during the localization process. }\end{array}$ & $\begin{array}{l}\text { High computational complexity is expected } \\
\text { due to the iterative nature of most ap- } \\
\text { proaches. Suboptimal solutions are expected } \\
\text { since approximation of the cost function is } \\
\text { usually required }\end{array}$ \\
\hline & Indirect & 209. 211 & $\begin{array}{l}\text { The range errors caused by the NLOS con- } \\
\text { dition are identified and compensated, and } \\
\text { then the compensated ranges are used in the } \\
\text { localization process. }\end{array}$ & $\begin{array}{l}\text { ML-based approaches are time consuming } \\
\text { to create a fingerprinting dataset. In scenar- } \\
\text { ios with high variability, the dataset may } \\
\text { require to be updated from time to time. }\end{array}$ \\
\hline
\end{tabular}

type mainly employ information related to the communication channel and/or transmit/received signals. In contrast, the approaches in the range-based type mainly employ the characteristics of the estimated ranges for distinguishing the LOS from the NLOS conditions.

Most of the recent approaches for identifying the NLOS conditions in the channel based type are based on supervised ML techniques in which the learning is based on an explicit known input/output (labels) relationship. Several approaches exploit well-known ML techniques such as convolutional neural network $(\mathrm{CNN})$, support vector machine (SVM), random forest (RF), artificial neural network (ANN), and adaptive boosting (AdaBoost) to distinguish the LOS and NLOS conditions. For instance, in [179] reference signals that allow the estimation of quality of the communication channel were employed to train a CNN for distinguishing the LOS and NLOS conditions. In [181], the AdaBoost was employed in which weak NLOS classifiers were combined to create a final stronger classifier. Interestingly, it was shown that combining the CNN with other ML techniques such as long short term memory can result in an improvement in the detection of the NLOS condition [183]. It was also shown that, by denoising the channel impulse response before training, the CNN can improve its classification accuracy although the improvement decreases as the number of convolutional layers increases [182]. Recently, a more sophisticated CNN, CapsNet [212], was modified to deal with signal classification in which signal waveform characteristics were converted in monochromatic images from which the CapsNet learns to distinguish between LOS and NLOS conditions [187]. In contrast to the previous works in which common channel responses were employed to distinguish the NLOS conditions, it was recently demonstrated that by including angular properties, the NLOS identification can be enhanced [184, 186].

The NLOS identification is also possible by employing characteristics of the ranges between TN and ANs. The idea behind is that the ranges in LOS are shorter than those in NLOS condition and consequently the residuals, i.e., differ- ences between ranges, are different as well. For instance, under the assumption that TOA and AOA estimates are readily available, [190] demonstrated that the ANs in an NLOS condition can be identified by a geometrical residual analysis in which a temporary position of the $\mathrm{TN}$ was first estimated for calculating the ranges between $\mathrm{TN}$ and ANs. The estimated ranges were employed to construct the geometrical residuals. Then, a threshold hypothesis test was employed for identifying the ANs in NLOS condition. Similarly, in [192] an averaged residual is employed in the detection hypothesis test. It was shown in [193] that, by combining multidimensional scaling [213] and quasi-accurate detection [214] techniques, the NLOS conditions can be distinguished. Although the approach proposed in [193] identifies the NLOS condition, it is limited to the case of only one AN with NLOS condition. An iterative method for NLOS identification was developed in [191], where the eigenvalues of the autocorrelation of the range errors composed of the noise measurement and NLOS bias were employed for distinguishing the NLOS condition. Recently, ranging between two ANs and TN together with the idea that Fresnel zones are different in LOS and NLOS conditions were employed to distinguish and mitigate the NLOS conditions [194]. Despite the simplicity of the approach, it shows a significant NLOS identification rate over other identification techniques such as CNN-based identification techniques [183, 187] in a limited scenario with few obstacles. Although the idea behind is simple, the NLOS identification is highly dependent on the detection threshold parameter, which in most cases is considered fixed for a specific scenario. Consequently, a high degradation could occur on the NLOS conditions identification in a highly dynamic scenario.

\section{2) Mitigation of Non-line of Sight}

Instead of using characteristics of the communication channel and range measurements for only identifying the NLOS condition, direct and indirect mitigation of the effect of NLOS on the localization accuracy is possible. While in the direct mitigation explicit identification of the NLOS condition is not required, in the indirect mitigation it is necessary. 
The direct mitigation can be achieved through several approaches. For instance, by employing robust statistics, the localization of a TN was conducted in a mixed LOS/NLOS scenarios where a weighted least square was adopted [195, 197]. The covariance information of the sample mean and sample median were exploited when the variance of the inliers was assumed to be known [195] and unknown [197]. More recently, under the assumption of known range error distribution, the TN localization was formulated in the variational Bayesian framework in which unknown parameters related to the LOS/NLOS conditions were incorporated and then treated as nuisance parameters [203]. Other approaches incorporate the bias error as an unknown parameter in the formulation of the localization problem, which usually is formulated in the maximum likelihood framework and solved with convex optimization techniques [196, 198-200, 204,-206]. Interestingly, it was shown recently that cooperative localization in which there exists cooperation between TNs is less sensitive to the NLOS conditions [204]. Therein, the NLOS error bias was bounded and employed as geometrical constraints in the formulation of the maximum likelihood estimation of the cooperative localization problem, which was relaxed by employing convex optimization techniques and then solved iteratively. Although popular, the NLOS mitigation approaches based on convex optimization are highly computationally demanding.

To reduce the computational complexity, some approaches based on the alternating direction method of multipliers (ADMM) were recently proposed [202, 208]. Simulation results demonstrate that approaches based on the ADMM provide similar NLOS mitigation performance to recent approaches based on convex optimization but only at low levels of noise and with a limited number of NLOS conditions. In contrast to other works in which statistical information of the communication channel is required, the work in [201] mitigates the NLOS bias error with reduced computation time by combining sparse techniques and Gaussian process (GP) regressor for a static TN. The case of moving TN was dealt in [207], where the echo state network together with GP regressor were employed to track a TN.

The indirect mitigation approaches improve the localization accuracy by first compensating the ranging errors caused by the NLOS condition. For instance, by employing fuzzy logic, NLOS conditions were identified and range errors compensated, and then the least affected ranges were employed in the localization process in [209]. Recently, the range errors caused by the NLOS conditions were compensated by remodeling the ranging model based on the time-of-flight for scenarios with known floor plans [210]. Although the approach proposed in [210] compensates the range error in a simple and elegant manner without requiring measurement campaigns, it is limited to certain scenarios with fixed known floor plans. In addition to this, only the NLOS conditions generated by the walls were considered. More recently, deep learning model together with graph optimization was employed for range compensation in NLOS conditions [211]. Therein, a raw channel impulse response was used instead of channel selected features in the learning process. In addition, the deep learning technique was optimized to reduce the computational complexity by employing pruning and quantization techniques. Interestingly, it was shown that pruning and quantization of the deep learning approach did not affect significantly the range compensation performance. Although most of the works only consider two conditions, i.e., LOS and NLOS, there are some works [215, 216] that divide the LOS and NLOS conditions further into pure LOS, in which only the DPCs exist; SoftNLOS, in which the DPC coexists with the IPCs; and hardNLOS, in which only IPCs exist. Considering these three conditions, the compensation for the range errors caused by the NLOS condition could be made more accurate since it was shown that the three conditions possess different deviation errors [216]. Further improvements can also be achieved by incorporating information from IMU [217].

\section{E. Discussion on the Recent Solutions}

\section{1) Discussion on Limited Number of Anchor Nodes}

On one hand, it is well documented that a cooperative localization scheme ameliorates successfully the limitation on the number of ANs. However, it is at the cost of increasing the communication overhead and computational complexity on the localization process that in some cases may run out the battery of sensor nodes, resulting in a counterproductive solution. Consequently, the cooperative localization should be performed discriminately by taking into account energyefficiency issues [218].

On the other hand, opportunistic approaches indeed seem interesting solutions but, for the sensor nodes to be able to exploit different types of SOP, they require highly complex front-ends with high bandwidth and high sampling capacity, which in most cases are restricted in WSNs. Additionally, special attention needs to be paid to the uncertainties of the positions of opportunistic reference nodes, since small deviations from their real positions may cause huge localization errors on the estimation of the unknown position of the TN [110]. Although the number of reference nodes can be increased and consequently the localization accuracy improved by employing SOP, it may also raise security issues. For instance, if a sensor node opportunistically considered is a malicious one, it may sabotage the localization process with disastrous consequences, e.g., a crash of UAVs [219].

\section{2) Discussion on the placement of Anchor Nodes}

Most of the recent approaches for optimizing/analyzing the placement of ANs are related/based on deterministic CRLB (GDOP), i.e, fixed scenarios with fixed positions and numbers of ANs and TNs. Such CRLB provides insights only on a specific scenario. Interestingly, an alternative metric called maximum angular separation (MAS) was introduced in [132], which provides insights in all positioning scenarios contrary to the CRLB. Additionally, the MAS metric also provides the probability that a TN is inside or outside of the convex hull formed with the ANs. Recently, based on a similar idea employed in [132], a random CRLB was introduced in [220], which posseses a probability distribution that provides insights of the localization accuracy in all possible scenarios that may arise within a WSN. The random CRLB may provide 
interesting insights not only on the optimal placement of ANs but also on how frequency reuse, processing demand in the sensor nodes, and ranging error affect the localization accuracy for a certain deployment [220].

Most of the approaches presented in Table II deal with the optimal placement of ANs under the assumption that only LOS is present. However, it is well known that the existence of NLOS is unavoidable especially in indoor scenarios. Consequently, NLOS conditions should also be considered for a more realistic optimization of the placement of ANs. One way to incorporate NLOS conditions is to include the uncertainty in the position of the sensor nodes [128]. In addition, the majority of works put aside other important aspects of WSNs such as connection/coverage reliability and deployment/maintenance cost. Besides securing the best possible deployment of sensors, securing connection/coverage reliability, battery lifetime, and minimum deployment/maintenance costs are also required for cost-effective WSNs. Such diverse considerations can be taken into account in the form of a multi objective problem for which solutions based on meta-heuristic algorithms may be an interesting option [221, 222].

\section{3) Discussion on Multipath Components}

Although the mitigation of MPCs is important, with the trend of increased communication bandwidth, the exploitation of MPCs is expected to be the general trend to follow. To take advantage of MPCs, the sensor nodes in WSNs need to distinguish between DPCs and IPCs. However, besides a high bandwidth, the sensor nodes need to incorporate high-end transceivers and large antenna arrays, which can be currently not plausible from an economical point of view. In spite of this, wireless technology is advancing at a great speed, so it is not difficult to expect that in the near future the capabilities of sensor nodes in WSN will provide the ability to distinguish DPC and IPCs at a reasonable price.

By exploiting the MPCs, the requirement of necessarily having two (in AOA based localization) or more ANs for finding the position of a $\mathrm{TN}$ in $\mathbb{R}^{2}$ will be avoided, and the localization with only one AN will be commercially possible. For instance, consider a 2D scenario depicted in Fig. 4 where a single TN (circle in dark orange) and AN (star in dark blue ) are located inside a room with four walls, i.e., $W 1, W 2, W 3$, and $W 4$. For simplicity, it is assumed that the AN and TN are surrounded only by the walls and only single bounce MPCs exist. In this simplified scenario, only one DPC and four IPCs are present where each IPC can be seen as a DPC from a VN (star in light blue). The position of each VN can be found by mirroring the AN at the corresponding wall. For example, the position of the $\mathrm{VN}_{1}$ can be found by mirroring the $\mathrm{AN}$ at $W 1$. Consequently, four VNs are created which have a huge implication in the localization accuracy since we move from a situation in which the localization of the TN was not possible due to a limited number of reference nodes to a situation where the localization can be achieved thanks to the increase of the number of reference nodes. A formal calculation of the position of the VNs can be found in [153, 159].

The exploitation of MPCs has recently caught the attention of the research community. An important issue is the cor-

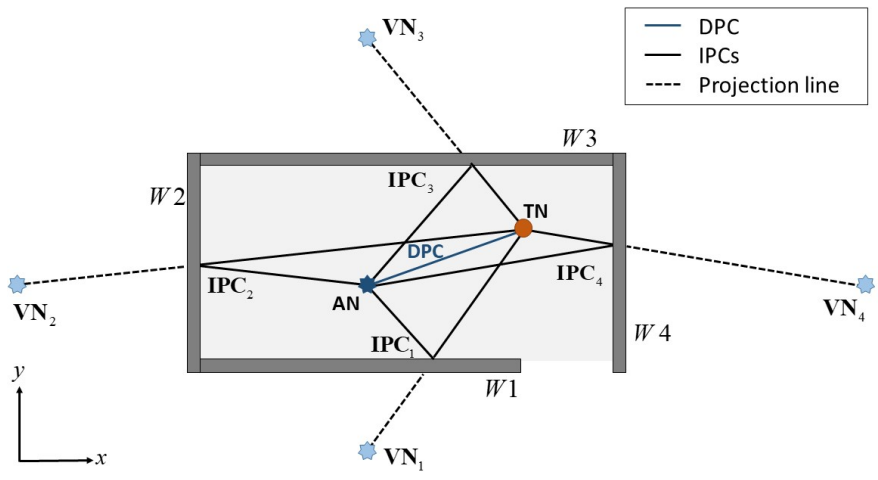

Fig. 4. A graphical representation of the exploitation of MPCs for generating VNs considering single bounces only.

rect association of the MPCs with their corresponding VNs. A wrong association may result in large localization errors [223]. Additionally, it is still an open issue to determine the conditions in which IPCs should be neglected or considered in the association process. Furthermore, mainly the specular with a single bounce IPCs are considered for creating the VNs. It could be beneficial to consider also multiple bounces and diffuse IPCs for creating more VNs to further enhance the localization accuracy.

\section{4) Discussion on Non-line of Sight}

To identify and mitigate NLOS conditions, several techniques have been proposed over the years, see Table IV However, due to recent milestones of artificial intelligence and data science, ML based approaches are gaining momentum not only for the LOS/NLOS identification but also for mitigating the bias error caused by the NLOS conditions. Nevertheless, such approaches require a time-consuming data labeling process that may become problematic in some highly dynamic scenarios in which the input/output relation needs to be updated each time the scenario varies. Additionally, such approaches are highly computationally demanding due to the requirement of a large amount of training and testing data. Fortunately, there are some ideas/techniques that can be used to ameliorate those limitations. To reduce the computational complexity, reduction of the size of the training data [185], network pruning, and quantization [211] techniques can be employed. Semi-supervised or completely unsupervised ML techniques may alleviate the requirement of explicit knowledge between the input and output [180, 188, 189].

Some WSNs with limited bandwidth such as the ones based on Wi-Fi provide limited information for identification and mitigation of NLOS conditions. This may be the reason why the majority of the works devoted to the identification and mitigation of NLOS employ different technologies with higher bandwidth such as the UWB technology, see Table IV] Having a larger bandwidth results in a finer resolution of the received signal which contributes to a finer distinction between the LOS and NLOS conditions. Nevertheless, the limitation in bandwidth may be alleviated by taking into account frequency and spatial diversity as in the cases of mitigation and exploitation of MPCs [224]. In addition, the incorporation of new measurements such as the ones obtained 
from IMUs may contribute to the same objective [217].

\section{Challenges And Perspective}

Although there have been considerable advances in dealing with the fundamental limitations, there still remain challenges that need to be addressed as presented in this section. Some perspectives that may help to solve such challenges will also be presented.

\section{A. Energy-Efficiency}

In WSNs, the sensor nodes are expected to have years of battery life hence their computational capabilities are usually restricted. This is due to the fact that sensor nodes need to satisfy certain conditions such as low price and low power consumption, especially in large deployments. If some sensor nodes increase their capabilities (e.g., ability to detect and discriminate more MPCs and NLOS conditions) it will require higher energy consumption to process the data, which may run out their batteries. If the battery runs out, the capabilities of the WSNs will diminish to perform any entrusted task, e.g., localization of TNs. Most of the localization approaches have focused on increasing the localization accuracy without paying attention to energy consumption. For instance, cooperative localization, which increases not only the localization accuracy but also the resilience of the WSN to the NLOS condition [204], can dry out the battery of the sensor nodes due to the increase of interactions among the sensor nodes. Blind increase of the interaction of the sensor nodes may result in an inefficient way of increasing the localization accuracy. Therefore, to use efficiently the battery of the sensors, a careful selection of the sensor nodes that will participate in the localization process is required. To do so, approaches based on game theory may be interesting options [225].

\section{B. Low Computational Complexity}

Since the sensor nodes in WSNs are constrained by the life span of their batteries and computational capabilities, in general, all the applications require low computational and energy-efficient algorithms. Consequently, the algorithms for dealing with the practical fundamental limitations are required to have low computational complexity and provide high localization accuracy. Usually, there exists a trade-off between the computation complexity of the algorithms and the localization accuracy. For instance, algorithms based on linear least squares are known to be simple with a high computational efficiency, but with a poor localization accuracy at low SNR. On the contrary, algorithms based on semidefinite programming and/or second order cone programming are known to provide a higher localization accuracy, but at the cost of a higher computational demand [80]. Recently, localization algorithms based on meta heuristic approaches are getting considerable attention due to the fact that they provide a more reasonable trade-off between localization accuracy and computational complexity [226-228]. Based on these works, it seems plausible to expect that meta heuristic approaches could provide interesting results for dealing also with the practical limitations with a considerably low computational complexity.

\section{Security}

It has been reported that malicious attacks are increasing not only to the wired but also to the wireless networks [229]. In fact, WSNs to some extend are more susceptible to be compromised because wireless signals can be reached immediately within the communication range of a sensor node. When a certain sensor node is compromised, the attackers can manipulate relevant information for dealing with the fundamental limitations presented in Section III such as the location of sensor nodes and measurements, which could lead to poor localization accuracy with unfortunate results. Although there are several works addressing the security in localization problems [230-233], they mostly assume ideal scenarios without considering the availability of reference nodes, MPCs, and NLOS conditions. In a similar way, most of the works that deal with fundamental limitations assume that all the sensor nodes provide reliable information, e.g, their position. Approaches considering malicious attacks for dealing with such limitations presented in Section $\Pi \Pi$ are crucial for improving secure localization based services. Exploiting the collaboration of sensor nodes may be an interesting option for increasing security, since it is less affected by a limited number of ANs and also showed resilience to NLOS conditions [204, 234]. Another option is to exploit SOP since it may increase the security by diversifying signals that are employed in the localization process [110]. A promising technology, blockchain, is making its way into WSNs, and it is expected to improve further the security in WSNs [235].

\section{Data Collection for ML-based Techniques}

One of the main drawbacks of ML-based techniques in countering fundamental limitations and enhancing the localization accuracy is their scenario dependency. Such dependency includes not only the elements surrounding the sensor nodes but also the characteristics of the technology employed. For instance, if we want to locate three different assets, e.g., cellphone, laptop, and RFID tag, in the same scenario, fingerprinting datasets for each asset are required. In addition to this, changes in the WSN configuration, e.g, increasing the coverage of the sensors, may result in demining the previous fingerprinting datasets and hence new datasets for each asset will be required, which is expensive and timeconsuming. In short, ML techniques with less dependency on a specific scenario are more desirable. Such dependencies may be ameliorated by exploiting new paradigms in ML such as the self-supervised learning [236].

\section{E. Heterogeneous Sensors Combination}

The robustness of any localization technique to the limitations discussed in Section III can be improved by combining different signal-related measurements such as RSS, TOA, and AOA. For instance, it is well-known that localization techniques employing the RSS and AOA measurements simultaneously provide a better localization accuracy than those employing only one of the measurements. In addition, a localization technique can also exploit measurements obtained 
from other sensors such as IMUs and cameras. By a careful combination of measurements, the resilience to fundamental limitations can be improved resulting in a more robust localization technique. Since the incorporation of new measurements from new sensors and the combination of them can be achieved in different ways, investigating the optimal and cost-effective combination is still an open issue.

\section{CONCLUSION}

In this review paper, a general taxonomy of the theoretical fundamental limits in wireless sensor networks were presented. Besides the well-known Cramer-Rao lower bound, there are other theoretical bounds tighter than the Cramer-Rao lower bound at low, medium, and high levels of signal to noise ratio. The tighter bounds that can be employed for a better comparison of the performance of localization algorithms are presented not only when the unknown position of the target node and other modeling parameters are considered deterministic but also when they are considered random and even hybrid (deterministic and random).

Fundamental practical limitations were also presented, where we paid special attention to the general limitations: the availability of reference nodes, placement of sensor nodes, multipath propagation, and non-line of sight. State-of-the-art solutions were presented for each of the general limitations. For increasing the availability of reference nodes in the localization process, two main approaches can be employed: cooperation of sensor nodes and exploitation of signals of opportunity in which opportunistically found signals together with their source position (known or unknown) information can be exploited for increasing the number of reference nodes and consequently for improving the localization accuracy. For optimizing the placement of sensor nodes, most of the current techniques are based on minimizing the geometric dilution of precision which is closely related to the Cramer-Rao lower bound or related parameters, e.g., the Fisher information matrix. Another parameter based on the angular formation between the sensor nodes was pointed out to provide further insights compared to the Cramer-Rao lower bound based optimal placement techniques.

For dealing with the multi-path components, we have discussed two approaches: mitigation and exploitation. While the mitigation approaches focus on mitigating the effects of the multipath component at the transceiver, medium access control, and model levels; exploitation of multipath component turns around the table and embraces the multipath components, converting them from foe to friends, which is possible thanks to high bandwidth and high resolution in time and frequency domain. Under the assumption of high resolvability of multipath components, virtual nodes can be created for the main multipath components with huge implications on the localization side. It was shown that, even with only one reference node, the localization of a target node could be possible. Although the exploitation and creation of virtual nodes just started, it shows a promising future that may change drastically the localization area.

For dealing with the non-line of sight conditions, the stateof-the-art approaches were grouped into two categories: iden- tification and mitigation. In the identification category are the approaches that focus on finding the existence of connections between the sensor nodes with non-line of sight conditions. Once those connections are found, they can be removed from the localization process. In contrast, in the mitigation category are the approaches that mitigates the effects of the non-line of sight conditions on the localization process. These approaches were grouped into two types: direct and indirect mitigation. Recent approaches in the two categories are mainly based on machine learning techniques with enhanced performance. However, most of such approaches require a time-consuming labeling process that in a highly dynamic process is highly inefficient.

Although there have been considerable advances for dealing with the fundamental limitations, there are still open issues that need to be addressed when dealing with the fundamental limitations such as energy efficiency, computational complexity, security, and scenario/data dependency for machine learning based techniques.

\section{REFERENCES}

[1] T. Wen and P. Zhu, "5G: A technology vision," Huawei - White paper, Feb. 2014.

[2] H. Wang and A. O. Fapojuwo, "A survey of enabling technologies of low power and long range machineto-machine communications," IEEE Commun. Surveys, Tuts., vol. 19, no. 4, pp. 2621-2639, 4th Quart. 2017.

[3] R. Du, P. Santi, M. Xiao, A. V. Vasilakos, and C. Fischione, "The sensable city: A survey on the deployment and management for smart city monitoring," IEEE Commun. Surveys, Tuts., vol. 21, no. 2, pp. 1533-1560, 2nd Quart. 2018.

[4] A. Grogan, "Smart farming," Eng., Technol., vol. 7, no. 6, pp. 38-40, July 2012.

[5] C. Kulatunga, L. Shalloo, W. Donnelly, E. Robson, and S. Ivanov, "Opportunistic wireless networking for smart dairy farming," IT Prof., vol. 19, no. 2, pp. 16-23, Apr. 2017.

[6] F. Viani, M. Bertolli, and A. Polo, "Low-cost wireless system for agrochemical dosage reduction in precision farming," IEEE Sensors J., vol. 17, no. 1, pp. 5-6, Jan. 2016.

[7] G. Ding, Q. Wu, L. Zhang, Y. Lin, T. A. Tsiftsis, and Y.D. Yao, "An amateur drone surveillance system based on the cognitive internet of things," IEEE Commun. Mag., vol. 56, no. 1, pp. 29-35, Jan. 2018.

[8] L. Kunze, N. Hawes, T. Duckett, M. Hanheide, and T. Krajník, "Artificial intelligence for long-term robot autonomy: A survey," IEEE Robot., Autom. Lett., vol. 3, no. 4, pp. 4023-4030, Oct. 2018.

[9] N. Strodthoff, B. Göktepe, T. Schierl, C. Hellge, and W. Samek, "Enhanced machine learning techniques for early harq feedback prediction in 5G," IEEE J. Sel. Areas Commun., vol. 37, no. 11, pp. 2573-2587, Nov. 2019.

[10] F. Zeng, C. Wang, and S. S. Ge, "A survey on visual navigation for artificial agents with deep reinforcement 
learning," IEEE Access, vol. 8, pp. 135426-135442, Aug. 2020.

[11] S. Chung, A. A. Paranjape, P. Dames, S. Shen, and V. Kumar, "A survey on aerial swarm robotics," IEEE Trans. Robot., vol. 34, no. 4, pp. 837-855, Aug. 2018.

[12] J. Li, Z. Li, F. Chen, A. Bicchi, Y. Sun, and T. Fukuda, "Combined sensing, cognition, learning, and control for developing future neuro-robotics systems: A survey," IEEE Trans. Cogn., Developmental Syst., vol. 11, no. 2, pp. 148-161, June 2019.

[13] S. Wu, S. Zhang, and D. Huang, "A TOA-based localization algorithm with simultaneous NLOS mitigation and synchronization error elimination," IEEE Sensors Lett., vol. 3, no. 3, pp. 1-4, Mar. 2019.

[14] W. Xiong and H. C. So, "TOA-based localization with NLOS mitigation via robust multidimensional similarity analysis," IEEE Signal Process. Lett., vol. 26, no. 9, pp. 1334-1338, Sep. 2019.

[15] C. Wu and J. Elangage, "Multiemitter two-dimensional angle-of-arrival estimator via compressive sensing," IEEE Trans. Aerosp., Electron. Syst., vol. 56, no. 4, pp. 2884-2895, Sep. 2019.

[16] Y. Zheng, M. Sheng, J. Liu, and J. Li, "Exploiting AoA estimation accuracy for indoor localization: A weighted AoA-based approach," IEEE Wireless Commun. Lett., vol. 8, no. 1, pp. 65-68, Feb. 2018.

[17] A. Zanella, "Best practice in RSS measurements and ranging," IEEE Commun. Surveys, Tuts., vol. 18, no. 4, pp. 2662-2686, 4th Quart. 2016.

[18] L. A. Caceres-Najarro, I. Song, and K. Kim, "Differential evolution with opposition and redirection for source localization using RSS measurements in wireless sensor networks," IEEE Trans. Autom. Sci., Eng., vol. 17, no. 4, pp. 1736-1747, Oct. 2020.

[19] X. Tian et al., "Performance analysis of Wi-Fi indoor localization with channel state information," IEEE Trans. Mobile Comput., vol. 18, no. 8, pp. 1870-1884, Aug. 2019.

[20] Z. Wu, Q. Xu, J. Li, C. Fu, Q. Xuan, and Y. Xiang, "Passive indoor localization based on CSI and naive bayes classification," IEEE Trans. Syst., Man, Cybern., Syst., vol. 48, no. 9, pp. 1566-1577, Sep. 2017.

[21] D. Plets, W. Deprez, J. Trogh, L. Martens, and W. Joseph, "Joint received signal strength, angle-ofarrival, and time-of-flight positioning," in Proc. Eur. Conf. Antennas Propag., Krakow, Poland, pp. 1-5, Mar. 2019.

[22] W. Li, L. Wang, M. Xiao, Y. Li, and H. Zhang, "Closed form solution for 3D localization based on joint RSS and AOA measurements for mobile communications," IEEE Access, vol. 8, pp. 12632-12643, Dec. 2019.

[23] C. Laoudias et al., "A survey of enabling technologies for network localization, tracking, and navigation," IEEE Commun. Surveys, Tuts., vol. 20, no. 4, pp. $3607-$ 3644, 4th Quart. 2018.

[24] S. Kekki et al., "MEC in 5G networks," ETSI white paper, vol. 28, pp. 1-28, June 2018.

[25] R. M. Buehrer, H. Wymeersch, and R. M. Vaghefi,
"Collaborative sensor network localization: Algorithms and practical issues," Proc. IEEE, vol. 106, no. 6, pp. 1089-1114, June 2018.

[26] F. Zafari, A. Gkelias, and K. K. Leung, "A survey of indoor localization systems and technologies," IEEE Commun. Surveys, Tuts., vol. 21, no. 3, pp. 1089-1114, 3rd Quart. 2019.

[27] D. B. Ahmed, L. E. Diez, E. M. Diaz, and J. J. G. Domingues, "A survey on test and evaluation methodologies of pedestrian localization systems," IEEE Sensors J., vol. 21, no. 3, pp. 479-491, Jan. 2020.

[28] S. Kuutti et al., "A survey of the state-of-the-art localization techniques and their potentials for autonomous vehicle applications," IEEE Internet Things J., vol. 5, no. 2, pp. 829-846, Apr. 2018.

[29] J. Liu, H. Liu, Y. Chen, Y. Wang, and C. Wang, "Wireless sensing for human activity: A survey," IEEE Commun. Surveys, Tuts., vol. 22, no. 3, pp. 1629-1645, 3rd Quart. 2020.

[30] Y. Li, Y. Zhuang, X. Hu, Z. Gao, J. Hu, L. Chen, Z. He, L. Pei, K. Chen, M. Wang et al., "Toward locationenabled IoT (LE-IoT): IoT positioning techniques, error sources, and error mitigation," IEEE Internet Things $J$., vol. 8, no. 6, pp. 4035-4062, Mar. 2021.

[31] S. M. Kay, Fundamentals of Statistical Signal Processing. Upper Saddle River, NJ: Prentice Hall PTR, 1993.

[32] V. M. Chiriac, Q. He, A. M. Haimovich, and R. S. Blum, "Ziv-Zakai bound for joint parameter estimation in MIMO radar systems," IEEE Trans. Signal Process., vol. 63, no. 18, pp. 4956-4968, Sep. 2015.

[33] Y. C. Eldar, "Minimum variance in biased estimation: Bounds and asymptotically optimal estimators," IEEE Trans. Signal Process., vol. 52, no. 7, pp. 1915-1930, July 2004.

[34] M. Fréchet, "Sur l'extension de certaines évaluations statistiques au cas de petits échantillons," Rev. Int. Statist. Inst., vol. 11, no. 3, pp. 182-205, Jan. 1943.

[35] G. Darmois, "Sur les limites de la dispersion de certaines estimations," Rev. Int. Statist. Inst., vol. 13, no. 1, pp. 9-15, Jan. 1945.

[36] C. R. Rao, "Minimum variance and the estimation of several parameters," Math. Proc. Cambridge Philosoph. Soc., vol. 43, no. 2, pp. 280-283, Apr. 1947.

[37] Z. Wang, H. Zhang, T. Lu, and T. A. Gulliver, "Cooperative RSS-based localization in wireless sensor networks using relative error estimation and semidefinite programming," IEEE Trans. Veh. Technol., vol. 68, no. 1, pp. 483-497, Jan. 2019.

[38] H. L. Van Trees, Detection, Estimation, and Modulation Theory, Part I: Detection, Estimation, and Linear Modulation Theory. New York, NY, USA, John Wiley \& Sons, 2004.

[39] A. O. Hero, J. A. Fessler, and M. Usman, "Exploring estimator bias-variance tradeoffs using the uniform CR bound," IEEE Trans. Signal Process., vol. 44, no. 8, pp. 2026-2041, Aug. 1996.

[40] Y. C. Eldar, "Uniformly improving the Cramér-Rao bound and maximum-likelihood estimation," IEEE 
Trans. Signal Process., vol. 54, no. 8, pp. 2943-2956, Aug. 2006.

[41] — - "MSE bounds with affine bias dominating the cramér-Rao bound," IEEE Trans. Signal Process., vol. 56, no. 8, pp. 3824-3836, Aug. 2008.

[42] B. Cernuschi-Frias, F. Gama, and D. Casaglia, "Deepest minimum criterion for biased affine estimation," IEEE Trans. Signal Process., vol. 62, no. 9, pp. 2437-2449, May 2014.

[43] L. Bacharach, C. Fritsche, U. Orguner, and E. Chaumette, "Some results on tighter bayesian lower bounds on the mean-square error," arXiv preprint arXiv:1907.09509, July 2019.

[44] D. Arora and M. McGuire, "Lower bounds on mobile terminal localisation in an urban area," IET Commun., vol. 5, no. 9, pp. 1182-1191, June. 2011.

[45] T. Young and R. Westerberg, "Error bounds for stochastic estimation of signal parameters," IEEE Trans. Inf. Theory, vol. 17, no. 5, pp. 549-557, Sep. 1971.

[46] Z. Ben-Haim and Y. C. Eldar, "A lower bound on the bayesian MSE based on the optimal bias function," IEEE Trans. Inf. Theory, vol. 55, no. 11, pp. 51795196, Nov. 2009.

[47] Y. Rockah and P. M. Schultheiss, "Array shape calibration using sources in unknown locations-Part I: Far-field sources," IEEE Trans. Acoust., Speech, Signal Process., vol. 35, no. 3, pp. 286-299, Mar. 1987.

[48] Y. Noam and H. Messer, "Notes on the tightness of the hybrid Cramér-Rao lower bound," IEEE Trans. Signal Process., vol. 57, no. 6, pp. 2074-2084, June 2009.

[49] F. Gini and R. Reggiannini, "On the use of CramerRao-like bounds in the presence of random nuisance parameters," IEEE Trans. Commun., vol. 48, no. 12, pp. 2120-2126, Dec. 2000.

[50] E. W. Barankin, "Locally best unbiased estimates," Ann. Math. Statist., vol. 20, no. 4, pp. 477-501, Dec. 1949.

[51] R. McAulay and E. Hofstetter, "Barankin bounds on parameter estimation," IEEE Trans. Inf. Theory, vol. 17, no. 6, pp. 669-676, Nov. 1971.

[52] J. S. Abel, "A bound on mean-square-estimate error," IEEE Trans. Inf. Theory, vol. 39, no. 5, pp. 1675-1680, Sep. 1993.

[53] I. Reuven and H. Messer, "On the effect of nuisance parameters on the threshold SNR value of the barankin bound," IEEE Trans. Signal Process., vol. 47, no. 2, pp. 523-527, Feb. 1999.

[54] E. Chaumette, J. Galy, A. Quinlan, and P. Larzabal, “A new Barankin bound approximation for the prediction of the threshold region performance of maximum likelihood estimators," IEEE Trans. Signal Process., vol. 56, no. 11, pp. 5319-5333, Nov. 2008.

[55] P. S. La-Rosa, A. Renaux, C. H. Muravchik, and A. Nehorai, "Barankin-type lower bound on multiple changepoint estimation," IEEE Trans. Signal Process., vol. 58, no. 11, pp. 5534-5549, Nov. 2010.

[56] S. S. Shalom and J. Tabrikian, "Efficient computation of MSE lower bounds via matching pursuit," IEEE Signal Process. Lett., vol. 24, no. 12, pp. 1798-1802, Dec.
2017.

[57] B. Bobrovsky and M. Zakai, "A lower bound on the estimation error for certain diffusion processes," IEEE Trans. Inf. Theory, vol. 22, no. 1, pp. 45-52, Jan. 1976.

[58] E. Nitzan, T. Routtenberg, and J. Tabrikian, "Bobrovsky-Zakai-type bound for periodic stochastic filtering," IEEE Signal Process. Lett., vol. 25, no. 10, pp. 1460-1464, Oct. 2018.

[59] C. Fritsche, U. Orguner, and F. Gustafsson, "BobrovskyZakai bound for filtering, prediction and smoothing of nonlinear dynamic systems," in Int. Conf. Inf. Fusion, Cambridge, UK, pp. 171-178, July 2018.

[60] I. Reuven and H. Messer, "A Barankin-type lower bound on the estimation error of a hybrid parameter vector," IEEE Trans. Inf. Theory, vol. 43, no. 3, pp. 1084-1093, May 1997.

[61] C. Ren, J. Galy, E. Chaumette, P. Larzabal, and A. Renaux, "Hybrid Barankin-Weiss-Weinstein bounds," IEEE Signal Process. Lett., vol. 22, no. 11, pp. 20642068, Nov. 2015.

[62] B.-Z. Bobrovsky, E. Mayer-Wolf, and M. Zakai, "Some classes of global Cramer-Rao bounds," Ann. Statist., vol. 15, no. 4, pp. 1421-1438, Dec. 1987.

[63] A. Weiss and E. Weinstein, "A lower bound on the mean-square error in random parameter estimation," IEEE Trans. Inf. Theory, vol. 31, no. 5, pp. 680-682, Sep. 1985.

[64] E. Weinstein and A. J. Weiss, "A general class of lower bounds in parameter estimation," IEEE Trans. Inf. Theory, vol. 34, no. 2, pp. 338-342, Mar. 1988.

[65] E. Chaumette, A. Renaux, and M. N. El Korso, "A class of Weiss-Weinstein bounds and its relationship with the Bobrovsky-Mayer-Wolf-Zakai bounds," IEEE Trans. Inf. Theory, vol. 63, no. 4, pp. 2226-2240, Apr. 2017.

[66] A. Renaux, P. Forster, P. Larzabal, C. D. Richmond, and A. Nehorai, "A fresh look at the bayesian bounds of the Weiss-Weinstein family," IEEE Trans. Signal Process., vol. 56, no. 11, pp. 5334-5352, Nov. 2008.

[67] C. Greiff, D. M. Núñez, M. A. González-Huici, and S. Brüggenwirth, "Adaptive transmission for radar arrays using Weiss-Weinstein bounds," IET Radar, Sonar, Navigation, vol. 12, no. 12, pp. 1390-1401, Dec. 2018.

[68] D. T. Vu, A. Renaux, R. Boyer, and S. Marcos, "Closedform expression of the Weiss-Weinstein bound for 3D source localization: The conditional case," in IEEE Sensor Array, Multichannel Signal Process. Workshop, Jerusalem, Israel, pp. 125-128, Oct. 2010.

[69] A. Renaux, "Weiss-Weinstein bound for data-aided carrier estimation," IEEE Trans. Signal Process., vol. 14, no. 4, pp. 283-286, Apr. 2007.

[70] L. Bacharach, M. N. El Korso, A. Renaux, and J. Y. Tourneret, "A hybrid lower bound for parameter estimation of signals with multiple change-points," IEEE Trans. Signal Process., vol. 67, no. 5, pp. 1267-1279, Mar. 2019.

[71] A. Mallat, S. Gezici, D. Dardari, C. Craeye, and L. Vandendorpe, "Statistics of the MLE and approximate upper 
and lower bounds-part I: Application to TOA estimation," IEEE Trans. Signal Process., vol. 62, no. 21, pp. 5663-5676, Nov. 2014.

[72] A. Gusi-Amigó, P. Closas, A. Mallat, and L. Vandendorpe, "Ziv-Zakai bound for direct position estimation," Navigation, vol. 65, no. 3, pp. 463-475, Apr. 2018.

[73] J. Ziv and M. Zakai, "Some lower bounds on signal parameter estimation," IEEE Trans. Inf. Theory, vol. 15, no. 3, pp. 386-391, May 1969.

[74] S. Bellini and G. Tartara, "Bounds on error in signal parameter estimation," IEEE Trans. Commun., vol. 22, no. 3, pp. 340-342, Mar. 1974.

[75] D. Chazan, M. Zakai, and J. Ziv, "Improved lower bounds on signal parameter estimation," IEEE Trans. Inf. Theory, vol. 21, no. 1, pp. 90-93, Jan. 1975.

[76] K. L. Bell, Y. Steinberg, Y. Ephraim, and H. L. V. Trees, "Extended Ziv-Zakai lower bound for vector parameter estimation," IEEE Trans. Inf. Theory, vol. 43, no. 2, pp. 624-637, Mar. 1997.

[77] A. G. Amigó, P. Closas, and L. Vandendorpe, "Mean square error bounds for parameter estimation under model misspecification," arXiv preprint arXiv:1511.03982, Dec. 2015.

[78] D. Khan and K. L. Bell, "Explicit Ziv-Zakai bound for analysis of DOA estimation performance of sparse linear arrays," Signal Process., vol. 93, no. 12, pp. 3449-3458, Mar. 2013.

[79] C. Ren, J. Galy, E. Chaumette, P. Larzabal, and A. Renaux, "A Ziv-Zakai type bound for hybrid parameter estimation," in Int. Conf. Acoust., Speech, Signal Process., Florence, Italy, pp. 4663-4667, May 2014.

[80] Y. Hu and G. Leus, "Robust differential received signal strength-based localization," IEEE Trans. Signal Process., vol. 65, no. 12, pp. 3261-3276, June 2017.

[81] I. Sharp, K. Yu, and Y. J. Guo, "GDOP analysis for positioning system design," IEEE Trans. Veh. Technol., vol. 58, no. 7, pp. 3371-3382, Sep. 2009.

[82] T. S. Rappaport, Wireless Communications: Principles and Practice, 2nd ed. Upper Saddle River, NJ, USA, Prentice hall PTR, 1996.

[83] E. Leitinger, P. Meissner, C. Rüdisser, G. Dumphart, and K. Witrisal, "Evaluation of position-related information in multipath components for indoor positioning," IEEE J. Sel. Areas Commun., vol. 33, no. 11, pp. 2313-2328, Nov. 2015.

[84] F. Tirado-Andrés and A. Araujo, "Performance of clock sources and their influence on time synchronization in wireless sensor networks," Int. J. Distrib. Sensor Net., vol. 15, no. 9, pp. 1-16, Sep. 2019.

[85] B. Liu, H. Chen, Z. Zhong, and H. V. Poor, "Asymmetrical round trip based synchronization-free localization in large-scale underwater sensor networks," IEEE Trans. Wireless Commun., vol. 9, no. 11, pp. 3532-3542, Nov. 2010.

[86] S. Li, M. Hedley, I. B. Collings, and D. Humphrey, "TDOA-based localization for semi-static targets in NLOS environments," IEEE Wireless Commun. Lett., vol. 4, no. 5, pp. 513-516, Oct. 2015.
[87] L. Patiño-Studencka, U. Batzer, and J. Thielecke, "Phase smoothing in a virtually synchronized pseudolite system using stochastic clock modelling," in Ubiquitous Positioning Indoor Navigation, Location Based Service, Kirkkonummi, Finland, pp. 1-5, Oct. 2010.

[88] M. Leng, F. Quitin, W. P. Tay, C. Cheng, S. G. Razul, and C. M. S. See, "Anchor-aided joint localization and synchronization using SOOP: Theory and experiments," IEEE Trans. Wireless Commun., vol. 15, no. 11, pp. 7670-7685, Nov. 2016.

[89] G. Lui, T. Gallagher, B. Li, A. G. Dempster, and C. Rizos, "Differences in RSSI readings made by different Wi-Fi chipsets: A limitation of WLAN localization," in Int. Conf. Localization, GNSS, Tampere, Finland, pp. 53-57, June 2011.

[90] B. Viel and M. Asplund, "Why is fingerprint-based indoor localization still so hard?" in IEEE Int. Conf. Pervasive Comput., Commun. Workshops, Budapest, Hungary, pp. 443-448, Mar. 2014.

[91] P. Tarrio, A. M. Bernardos, and J. R. Casar, "An RSS localization method based on parametric channel models," in Int. Conf. Sensor Technol., Appl., Valencia, Spain, pp. 265-270, Oct. 2007.

[92] R. W. Ouyang, A. K. S. Wong, and C. T. Lea, "Received signal strength-based wireless localization via semidefinite programming: Noncooperative and cooperative schemes," IEEE Trans. Veh. Technol., vol. 59, no. 3, pp. 1307-1318, Mar. 2010.

[93] S. Tomic, M. Beko, and R. Dinis, "RSS-based localization in wireless sensor networks using convex relaxation: Noncooperative and cooperative schemes," IEEE Trans. Veh. Technol., vol. 64, no. 5, pp. 20372050, May 2015.

[94] S. Chang, Y. Li, H. Wang, W. Hu, and Y. Wu, "RSSbased cooperative localization in wireless sensor networks via second-order cone relaxation," IEEE Access, vol. 6, pp. 54 097-54 105, Sep. 2018.

[95] Y. S. Meng and Y. H. Lee, "Measurements and characterizations of air-to-ground channel over sea surface at C-band with low airborne altitudes," IEEE Trans. Veh. Technol., vol. 60, no. 4, pp. 1943-1948, May 2011.

[96] X. Ge, R. Zi, H. Wang, J. Zhang, and M. Jo, "Multiuser massive MIMO communication systems based on irregular antenna arrays," IEEE Trans. Wireless Commun., vol. 15, no. 8, pp. 5287-5301, Aug. 2016.

[97] H. Steyskal and J. S. Herd, "Mutual coupling compensation in small array antennas," IEEE Trans. Antennas Propag., vol. 38, no. 12, pp. 1971-1975, Dec. 1990.

[98] C. L. Liu and P. P. Vaidyanathan, "Super nested arrays: Linear sparse arrays with reduced mutual coupling-part I: Fundamentals," IEEE Trans. Signal Process., vol. 64, no. 15, pp. 3997-4012, Aug. 2016.

[99] J. A. Peral-Rosado et al., "Whitepaper on new localization methods for $5 \mathrm{G}$ wireless systems and the internetof-things," IRACON - White paper, Apr. 2018.

[100] T. Yang, Y. Niu, and J. Yu, "Clock synchronization in wireless sensor networks based on bayesian estimation," IEEE Access, vol. 8, pp. 69683-69 694, Apr. 2020. 
[101] D. G. Enzer, D. W. Murphy, and E. A. Burt, "Allan deviation of atomic clock frequency-corrections: A new diagnostic tool for characterizing clock disturbances," IEEE Trans. Ultrason., Ferroelectr., Freq. Control, vol. 68, no. 7, pp. 2590-2601, July 2021.

[102] Z. He, Y. Li, L. Pei, R. Chen, and N. El Sheimy, "Calibrating multi-channel RSS observations for localization using Gaussian process," IEEE Wireless Commun. Lett., vol. 8, no. 4, pp. 1116-1119, Aug. 2019.

[103] F. C. Chen, J. F. Chen, Q. X. Chu, and M. J. Lancaster, "X-band waveguide filtering antenna array with nonuniform feed structure," IEEE Trans. Microw. Theory, Techn., vol. 65, no. 12, pp. 4843-4850, Dec. 2017.

[104] H. N. Chu and T. G. Ma, "An extended $4 \times 4$ butler matrix with enhanced beam controllability and widened spatial coverage," IEEE Trans. Microw. Theory, Techn., vol. 66, no. 3, pp. 1301-1311, Mar. 2018.

[105] H. Zhu, H. Sun, B. Jones, C. Ding, and Y. J. Guo, "Wideband dual-polarized multiple beam-forming antenna arrays," IEEE Trans. Antennas, Propag., vol. 67, no. 3, pp. 1590-1604, Mar. 2019.

[106] F. Zorzi and A. Zanella, "Opportunistic localization: Modeling and analysis," in IEEE Veh. Technol. Conf., Barcelona, Spain, pp. 1-5, Apr. 2009.

[107] A. Uchiyama, S. Fujii, K. Maeda, T. Umedu, H. Yamaguchi, and T. Higashino, "UPL: Opportunistic localization in urban districts," IEEE Trans. Mobile Comput., vol. 12, no. 5, pp. 1009-1022, May 2013.

[108] S. Misra, T. Ojha, and A. Mondal, "Game-theoretic topology control for opportunistic localization in sparse underwater sensor networks," IEEE Trans. Mobile Comput., vol. 14, no. 5, pp. 990-1003, May 2015.

[109] H. Simkovits, A. J. Weiss, and A. Amar, "Navigation by inertial device and signals of opportunity," Signal Process., vol. 131, pp. 280-287, Aug. 2017.

[110] Z. Z. M. Kassas, J. Khalife, K. Shamaei, and J. Morales, "I hear, therefore I know where I am: Compensating for GNSS limitations with cellular signals," IEEE Signal Process. Mag., vol. 34, no. 5, pp. 111-124, Sep. 2017.

[111] K. Shamaei and Z. M. Kassas, "A joint TOA and DOA acquisition and tracking approach for positioning with LTE signals," IEEE Trans. Signal Process., vol. 69, pp. 2689-2705, Mar. 2021.

[112] A. Young, J. Soli, and G. Hickman, "Self-localization technique for unmanned underwater vehicles using sources of opportunity and a single hydrophone," in OCEANS, Anchorage, AK, USA, pp. 1-6, Sep. 2017.

[113] C. M. Verlinden, J. Sarkar, B. D. Cornuelle, and W. A. Kuperman, "Determination of acoustic waveguide invariant using ships as sources of opportunity in a shallow water marine environment," J. Acoustical Soc. Amer., vol. 141, no. 2, pp. 102-107, Feb. 2017.

[114] A. H. Young, H. A. Harms, G. W. Hickman, J. S. Rogers, and J. L. Krolik, "Waveguide-invariant-based ranging and receiver localization using tonal sources of opportunity," IEEE J. Ocean. Eng., vol. 45, no. 2, pp. 631-644, Apr. 2020.

[115] J. Khalife and Z. M. Kassas, "Opportunistic UAV navigation with carrier phase measurements from asynchronous cellular signals," IEEE Trans. Aerosp., Electron. Syst., vol. 56, no. 4, pp. 3285-3301, Aug. 2020.

[116] K. Liu and X. Li, "Enhancing localization scalability and accuracy via opportunistic sensing," IEEE/ACM Trans. Netw., vol. 26, no. 3, pp. 1517-1530, June 2018.

[117] M. Page and T. L. Wickramarathne, "Enhanced situational awareness with signals of opportunity: RSS-based localization and tracking," in IEEE Intell. Transp. Syst. Conf., Auckland, New Zealand, pp. 3833-3838, Oct. 2019.

[118] N. Souli, R. Makrigiorgis, P. Kolios, and G. Ellinas, "Cooperative relative positioning using signals of opportunity and inertial and visual modalities," in IEEE Veh. Technol. Conf., Helsinki, Finland, pp. 1-7, Apr. 2021.

[119] C. Gao, G. Zhao, and H. Fourati, Cooperative Localization and Navigation: Theory, Research, and Practice. Boca Raton, FL, USA, CRC Press, 2019.

[120] J. J. Morales and Z. M. Kassas, "Tightly coupled inertial navigation system with signals of opportunity aiding," IEEE Trans. Aerosp., Electron. Syst., vol. 57, no. 3, pp. 1930-1948, June 2021.

[121] R. Kapoor, S. Ramasamy, A. Gardi, and R. Sabatini, "UAV navigation using signals of opportunity in urban environments: A review," Energy Procedia, vol. 110, pp. 377-383, Mar. 2017.

[122] S. Monica and G. Ferrari, "UWB-based localization in large indoor scenarios: Optimized placement of anchor nodes," IEEE Trans. Aerosp., Electro. Syst., vol. 51, no. 2, pp. 987-999, Apr. 2015.

[123] H. Sallouha, M. M. Azari, A. Chiumento, and S. Pollin, "Aerial anchors positioning for reliable RSS-based outdoor localization in urban environments," IEEE Wireless Commun. Lett., vol. 7, no. 3, pp. 376-379, June 2018.

[124] X. Fang and J. Li, "Frame theory for optimal sensor augmentation problem of AOA localization," IEEE Signal Process. Lett., vol. 25, no. 9, pp. 1310-1314, Nov. 2018.

[125] J. J. Khalife and Z. Z. M. Kassas, "Optimal sensor placement for dilution of precision minimization via quadratically constrained fractional programming," IEEE Trans. Aerosp., Electron. Syst., vol. 55, no. 4, pp. 2086-2096, Aug. 2019.

[126] O. Oshiga, O. Aina, A. Obadiah, A. Salihu, and G. Koyunlu, "Sensor placement for wireless localization," in Int. Conf. Electron., Comput., Comput., Abuja, Nigeria, pp. 1-6, Dec. 2019.

[127] M. Sadeghi, F. Behnia, and R. Amiri, "Optimal sensor placement for 2-D range-only target localization in constrained sensor geometry," IEEE Trans. Signal Process., vol. 68, pp. 2316-2327, Apr. 2020.

[128] S. Wang, S. Wang, W. Liu, and Y. Tian, "A study on the optimization nodes arrangement in UWB localization," Measurement, vol. 163, pp. 1-9, Oct. 2020.

[129] N. H. Nguyen, "Optimal geometry analysis for target localization with bayesian priors," IEEE Access, vol. 9, pp. 33419-33437, Mar. 2021. 
[130] S. Karimi-Bidhendi, J. Guo, and H. Jafarkhani, "Energy-efficient node deployment in heterogeneous two-tier wireless sensor networks with limited communication range," IEEE Trans. Wireless Commun., vol. 20, no. 1, pp. 40-55, Jan. 2021.

[131] X. Bo, A. A. Razzaqi, and G. Farid, "A review on optimal placement of sensors for cooperative localization of AUVs," J. Sensors, vol. 2019, pp. 1-12, July 2019.

[132] J. Schloemann, H. S. Dhillon, and R. M. Buehrer, "A tractable metric for evaluating base station geometries in cellular network localization," IEEE Wireless Commun. Lett., vol. 5, no. 2, pp. 140-143, Apr. 2016.

[133] Y. Y. Chen, S. P. Huang, T. W. Wu, W. T. Tsai, C. Y. Liou, and S. G. Mao, "UWB system for indoor positioning and tracking with arbitrary target orientation, optimal anchor location, and adaptive NLOS mitigation," IEEE Trans. Veh. Technol., vol. 69, no. 9, pp. 9304-9314, Sep. 2020.

[134] R. O. Schmidt, "A new approach to geometry of range difference location," IEEE Trans. Aerosp., Electron. Syst., vol. 8, no. 6, pp. 821-835, Nov. 1972.

[135] J. Kovacevic and A. Chebira, "Life beyond bases: The advent of frames (part I)," IEEE Signal Process. Mag., vol. 24, no. 4, pp. 86-104, July 2007.

[136] S. Lee, E. S. Lohan, and S. Kim, "Array-based GNSS signal tracking with a reduced state signal model," IEEE Trans. Aerosp. Electron. Syst., vol. 52, no. 3, pp. 12671283, June 2016.

[137] K. Zhang, B. Li, X. Zhu, H. Chen, and G. Sun, "Multipath detection based on single orthogonal dual linear polarized GNSS antenna," GPS Solutions, vol. 21, no. 3, pp. 1203-1211, Feb. 2017.

[138] L. Xie, X. Cui, S. Zhao, and M. Lu, "Mitigating multipath bias using a dual-polarization antenna: Theoretical performance, algorithm design, and simulation," Sensors, vol. 17, no. 2, pp. 1-23, Feb. 2017.

[139] M. Golmohamadi, A. Narbudowicz, and J. Frolik, "Mitigating indoor channels with quad-polarization diversity," IEEE Antennas Wireless Prop. Lett., vol. 18, no. 6, pp. 1199-1202, June 2019.

[140] B. Zhou, A. Liu, and V. Lau, "Successive localization and beamforming in 5G mmWave MIMO communication systems," IEEE Trans. Signal Process., vol. 67, no. 6, pp. 1620-1635, Mar. 2019.

[141] H. Chung, J. Kang, H. Kim, Y. M. Park, and S. Kim, "Adaptive beamwidth control for mmWave beam tracking," IEEE Comm. Lett., vol. 25, no. 1, pp. 137-141, Jan. 2021.

[142] Z. Chen, Z. Li, X. Zhang, G. Zhu, Y. Xu, J. Xiong, and $X$. Wang, "AWL: Turning spatial aliasing from foe to friend for accurate WiFi localization," in Proc. 13th Int. Conf. Emerg. Netw. Experiments, Technol., Seoul, Korea, pp. 238-250, Dec. 2017.

[143] V. Cantón-Paterna, A. Calveras-Auge, J. ParadellsAspas, and M. A. Perez-Bullones, "A bluetooth low energy indoor positioning system with channel diversity, weighted trilateration and Kalman filtering," Sensors, vol. 17, no. 12, pp. 1-32, Dec. 2017.
[144] E. Yuan, W. Qi, P. Liu, L. Wei, and L. Chen, "Ranging method for navigation based on high-speed frequencyhopping signal," IEEE Access, vol. 6, pp. 4308-4320, Feb. 2018.

[145] X. Ouyang, Q. He, Y. Yang, and Q. Wan, "TDOA/FDOA estimation algorithm of frequencyhopping signals based on CAF coherent integration," IET Commun., vol. 14, no. 2, pp. 331-336, Jan. 2020.

[146] J. Ma and Y. Yang, "A generalized anti-interference low-ambiguity dual-frequency multiplexing modulation based on the frequency-hopping technique," IEEE Access, vol. 8, pp. 95 288-95 300, June 2020.

[147] J. Lesouple, T. Robert, M. Sahmoudi, J. Y. Tourneret, and W. Vigneau, "Multipath mitigation for GNSS positioning in an urban environment using sparse estimation," IEEE Trans. Intell. Transp. Syst., vol. 20, no. 4, pp. 1316-1328, Apr. 2019.

[148] H. Qin, X. Xue, and Q. Yang, "GNSS multipath estimation and mitigation based on particle filter," IET Radar, Sonar, Navigation, vol. 13, no. 9, pp. 1588-1596, May 2019.

[149] S. K. Meghani, M. Asif, F. Awin, and K. Tepe, "Empirical based ranging error mitigation in IR-UWB: A fuzzy approach," IEEE Access, vol. 7, pp. 33 686-33 697, Mar. 2019.

[150] Y. Zhang, Y. Ma, X. Miao, S. Zhang, and B. Wang, "Multipath mitigation algorithm for multifrequencybased ranging via convex relaxation in passive UHF RFID," IEEE Internet Things J., vol. 6, no. 1, pp. 744752, Feb. 2019.

[151] C. Cheng, J. Y. Tourneret, and X. Lu, "A RaoBlackwellized particle filter with variational inference for state estimation with measurement model uncertainties," IEEE Access, vol. 8, pp. 55 665-55 675, Mar. 2020.

[152] P. Zhang, D. Li, J. Zhao, and J. Cheng, "Multipath mitigation in GNSS positioning by the dual-path compression estimation," IEEE Sensors J., vol. 20, no. 6, pp. 3087-3100, Mar. 2020.

[153] C. Gentner, T. Jost, W. Wang, S. Zhang, A. Dammann, and U. C. Fiebig, "Multipath assisted positioning with simultaneous localization and mapping," IEEE Trans. Wireless Commun., vol. 15, no. 9, pp. 6104-6117, Sep. 2016.

[154] B. Großwindhager, M. Rath, J. Kulmer, M. S. Bakr, C. A. Boano, K. Witrisal, and K. Römer, "SALMA: UWB-based single-anchor localization system using multipath assistance," in Proc. 16th ACM Conf. Embedded Netw. Sensor Syst., Shenzhen, China, pp. 132-144, Nov. 2018.

[155] X. Li, E. Leitinger, M. Oskarsson, K. Åström, and F. Tufvesson, "Massive MIMO-based localization and mapping exploiting phase information of multipath components," IEEE Trans. Wireless Commun., vol. 18, no. 9, pp. 4254-4267, Sep. 2019.

[156] R. Mendrzik, F. Meyer, G. Bauch, and M. Z. Win, "Enabling situational awareness in millimeter wave massive MIMO systems,' IEEE J. Sel. Topics Signal Process., 
vol. 13, no. 5, pp. 1196-1211, Sep. 2019.

[157] Y. Ge, F. Wen, L. Svensson, S. Kim, and H. Wymeersch, "Exploiting diffuse multipath in 5G SLAM," in Proc. IEEE Global Commun. Conf., Taipei, Taiwan, pp. 1-6, Dec. 2020.

[158] H. Kim, K. Granström, L. Gao, G. Battistelli, S. Kim, and $\mathrm{H}$. Wymeersch, "5G mmWave cooperative positioning and mapping using multi-model phd filter and map fusion," IEEE Trans. Wireless Commun., vol. 19, no. 6, pp. 3782-3795, June 2020.

[159] X. Chu, Z. Lu, D. Gesbert, L. Wang, and X. Wen, "Vehicle localization via cooperative channel mapping," IEEE Trans. Veh. Technol., vol. 70, no. 6, June 2021.

[160] C. Chen, Y. Chen, Y. Han, H. Q. Lai, and K. J. R. Liu, "Achieving centimeter-accuracy indoor localization on WiFi platforms: A frequency hopping approach," IEEE Internet Things J., vol. 4, no. 1, pp. 111-121, Feb. 2017.

[161] M. Sousa and R. S Thomä, "Enhancement of localization systems in NLOS urban scenario with multipath ray tracing fingerprints and machine learning," Sensors, vol. 18, no. 11, pp. 1-30, Nov. 2018.

[162] J. Fan, S. Chen, X. Luo, Y. Zhang, and G. Y. Li, "A machine learning approach for hierarchical localization based on multipath MIMO fingerprints," IEEE Commun. Lett., vol. 23, no. 10, pp. 1765-1768, Oct. 2019.

[163] C. Li, S. De Bast, E. Tanghe, S. Pollin, and W. Joseph, "Towards fine-grained indoor localization based on massive MIMO-OFDM system: Perspective of multipath components," arXiv preprint arXiv:2103.14863, Mar. 2021.

[164] T. Manabe, Y. Miura, and T. Ihara, "Effects of antenna directivity and polarization on indoor multipath propagation characteristics at $60 \mathrm{GHz}$," IEEE J. Sel. Areas Commun., vol. 14, no. 3, pp. 441-448, Apr. 1996.

[165] B. Wang, Y. Wang, X. Qiu, and Y. Shen, "BLE localization with polarization sensitive array," IEEE Wireless Commun. Lett., vol. 10, no. 5, pp. 1014-1017, May 2021.

[166] A. Guerra, F. Guidi, and D. Dardari, "Single-anchor localization and orientation performance limits using massive arrays: MIMO vs. beamforming," IEEE Trans. Wireless Commun., vol. 17, no. 8, pp. 5241-5255, Aug. 2018.

[167] J. A. del Peral-Rosado, J. A. López-Salcedo, and G. Seco-Granados, "Impact of frequency-hopping NBIoT positioning in $4 \mathrm{G}$ and future $5 \mathrm{G}$ networks," in IEEE Int. Conf. Commun. Workshops, Paris, France, pp. 815820, May 2017.

[168] A. Zanella and A. Bardella, "RSS-based ranging by multichannel RSS averaging," IEEE Wireless Commun. Lett., vol. 3, no. 1, pp. 10-13, Feb. 2014.

[169] Z. He, Y. Li, L. Pei, R. Chen, and N. El-Sheimy, "Calibrating multi-channel RSS observations for localization using gaussian process," IEEE Wireless Commun. Lett., vol. 8, no. 4, pp. 1116-1119, Aug. 2019.

[170] X. Fang and L. Chen, "An optimal multi-channel trilateration localization algorithm by radio-multipath multiobjective evolution in RSS-ranging-based wireless sen- sor networks," Sensors, vol. 20, no. 6, pp. 1-14, Mar. 2020.

[171] A. Giremus, J. Y. Tourneret, and V. Calmettes, "A particle filtering approach for joint detection/estimation of multipath effects on GPS measurements," IEEE Trans. Signal Process., vol. 55, no. 4, pp. 1275-1285, Apr. 2007.

[172] A. F. Molisch et al., "A comprehensive standardized model for ultrawideband propagation channels," IEEE Trans. Antennas, Propag., vol. 54, no. 11, pp. 31513166, Nov. 2006.

[173] J. Kulmer, E. Leitinger, S. Grebien, and K. Witrisal, "Anchorless cooperative tracking using multipath channel information," IEEE Trans. Wireless Commun., vol. 17, no. 4, pp. 2262-2275, Apr. 2018.

[174] Y. Ma, B. Wang, S. Pei, Y. Zhang, S. Zhang, and J. Yu, "An indoor localization method based on AOA and PDOA using virtual stations in multipath and NLOS environments for passive UHF RFID," IEEE Access, vol. 6, pp. 31772-31 782, June 2018.

[175] R. Mendrzik, H. Wymeersch, G. Bauch, and Z. AbuShaban, "Harnessing NLOS components for position and orientation estimation in 5G millimeter wave MIMO," IEEE Trans. Wireless Commun., vol. 18, no. 1, pp. 93-107, Jan. 2019.

[176] F. Wen and H. Wymeersch, "5G synchronization, positioning, and mapping from diffuse multipath," IEEE Wireless Commun. Lett., vol. 10, no. 1, pp. 43-47, Jan. 2021.

[177] F. Wen, J. Kulmer, K. Witrisal, and H. Wymeersch, "5G positioning and mapping with diffuse multipath," IEEE Trans. Wireless Commun., vol. 20, no. 2, pp. 1164 1174, Feb. 2021.

[178] A. Nessa, B. Adhikari, F. Hussain, and X. N. Fernando, "A survey of machine learning for indoor positioning," IEEE Access, vol. 8, pp. 214 945-214 965, Nov. 2020.

[179] T. Zeng, Y. Chang, Q. Zhang, M. Hu, and J. Li, "CNNbased LOS/NLOS identification in 3-D massive MIMO systems," IEEE Commun. Lett., vol. 22, no. 12, pp. 2491-2494, Oct. 2018.

[180] J. Fan and A. S. Awan, "Non-line-of-sight identification based on unsupervised machine learning in ultra wideband systems," IEEE Access, vol. 7, pp. 32 464-32 471, Mar. 2019.

[181] Y. Zhu, W. Xia, F. Yan, and L. Shen, "NLOS identification via AdaBoost for wireless network localization," IEEE Commun. Lett., vol. 23, no. 12, pp. 2234-2237, Dec. 2019.

[182] C. Jiang, S. Chen, Y. Chen, D. Liu, and Y. Bo, "An UWB channel impulse response de-noising method for NLOS/LOS classification boosting," IEEE Commun. Lett., vol. 24, no. 11, pp. 2513-2517, Nov. 2020.

[183] C. Jiang, J. Shen, S. Chen, Y. Chen, D. Liu, and Y. Bo, "UWB NLOS/LOS classification using deep learning method," IEEE Commun. Lett., vol. 24, no. 10, pp. 2226-2230, Oct. 2020.

[184] C. Huang, A. F. Molisch, R. He, R. Wang, P. Tang, B. Ai, and Z. Zhong, "Machine learning-enabled 
LOS/NLOS identification for MIMO systems in dynamic environments," IEEE Trans. Wireless Commun., vol. 19, no. 6, pp. 3643-3657, June 2020.

[185] V. B. Vales, T. Domínguez-Bolaño, C. J. Escudero, and J. A. García-Naya, "Using the power delay profile to accelerate the training of neural network-based classifiers for the identification of LOS and NLOS UWB propagation conditions," IEEE Access, vol. 8, pp. 220 205-220 214, Dec. 2020.

[186] Q. Zheng, R. He, B. Ai, C. Huang, W. Chen, Z. Zhong, and H. Zhang, "Channel non-line-of-sight identification based on convolutional neural networks," IEEE Wireless Commun. Lett., vol. 9, no. 9, pp. 1500-1504, Sep. 2020.

[187] Z. Cui, T. Liu, S. Tian, R. Xu, and J. Cheng, "Nonline-of-sight identification for UWB positioning using capsule networks," IEEE Commun. Lett., vol. 24, no. 10, pp. 2187-2190, Oct. 2020.

[188] C. H. Park and J. H. Chang, "WLS localization using skipped filter, Hampel filter, bootstrapping and Gaussian mixture EM in LOS/NLOS conditions," IEEE Access, vol. 7, pp. 35 919-35 928, Mar. 2019.

[189] T. Wang, K. Hu, Z. Li, K. Lin, J. Wang, and Y. Shen, "A semi-supervised learning approach for UWB ranging error mitigation," IEEE Wireless Commun. Lett., vol. 10, no. 3, pp. 688-691, Mar. 2021.

[190] J. Hua, Y. Yin, W. Lu, Y. Zhang, and F. Li, "NLOS identification and positioning algorithm based on localization residual in wireless sensor networks," Sensors, vol. 18, no. 9, pp. 1-12, Sep. 2018.

[191] A. A. Momtaz, F. Behnia, R. Amiri, and F. Marvasti, "NLOS identification in range-based source localization: Statistical approach," IEEE Sensors J., vol. 18, no. 9, pp. 3745-3751, May 2018.

[192] L. Cheng, Y. Li, Y. Wang, Y. Bi, L. Feng, and M. Xue, "A triple-filter NLOS localization algorithm based on fuzzy C-means for wireless sensor networks," Sensors, vol. 19, no. 5, pp. 1-24, Mar. 2019.

[193] Y. Zhu, T. Ma, Z. Li, D. Sun, X. Sun, X. Zhao, and F. Hu, "NLOS identification and correction based on multidimensional scaling and quasi-accurate detection," IEEE Access, vol. 7, pp. 53 977-53 987, May 2019.

[194] M. Dong, "A low-cost NLOS identification and mitigation method for UWB ranging in static and dynamic environments," IEEE Commun. Lett., vol. 25, no. 7, pp. 2420-2424, July 2021.

[195] C. H. Park and J. H. Chang, "Robust time-of-arrival source localization employing error covariance of sample mean and sample median in line-of-sight/non-lineof-sight mixture environments," EURASIP J. Advances Signal Process., vol. 89, no. 1, pp. 1-11, Aug. 2016.

[196] R. M. Vaghefi and R. M. Buehrer, "Cooperative source node tracking in non-line-of-sight environments," IEEE Trans. Mobile Comput., vol. 16, no. 5, pp. 1287-1299, May 2017.

[197] C. H. Park and J. H. Chang, "WLS localization using skipped filter, Hampel filter, bootstrapping and Gaussian mixture EM in LOS/NLOS conditions," IEEE Access, vol. 7, pp. 35 919-35 928, Apr. 2019.
[198] S. Gao, F. Zhang, and G. Wang, "NLOS error mitigation for TOA-based source localization with unknown transmission time," IEEE Sensors J., vol. 17, no. 12, pp. 3605-3606, June 2017.

[199] Z. Su, G. Shao, and H. Liu, "Semidefinite programming for NLOS error mitigation in TDOA localization," IEEE Commun. Lett., vol. 22, no. 7, pp. 1430-1433, July 2018.

[200] H. Chen, G. Wang, and N. Ansari, "Improved robust TOA-based localization via NLOS balancing parameter estimation," IEEE Trans. Veh. Technol., vol. 68, no. 6, pp. 6177-6181, June 2019.

[201] X. Yang, "NLOS mitigation for UWB localization based on sparse pseudo-input Gaussian process," IEEE Sensors J., vol. 18, no. 10, pp. 4311-4316, May 2018.

[202] W. Xiong and H. C. So, "TOA-based localization with NLOS mitigation via robust multidimensional similarity analysis," IEEE Signal Process. Lett., vol. 26, no. 9, pp. 1334-1338, Sep. 2019.

[203] Y. Li, S. Ma, G. Yang, and K. K. Wong, "Robust localization for mixed LOS/NLOS environments with anchor uncertainties," IEEE Trans. Commun., vol. 68, no. 7, pp. 4507-4521, July 2020.

[204] Y. Wang, K. Gu, Y. Wu, W. Dai, and Y. Shen, "NLOS effect mitigation via spatial geometry exploitation in cooperative localization," IEEE Trans. Wireless Commun., vol. 19, no. 9, pp. 6037-6049, Sep. 2020.

[205] Y. Sun, S. Yang, G. Wang, and H. Chen, "Robust RSS-based source localization with unknown model parameters in mixed LOS/NLOS environments," IEEE Trans. Veh. Technol., vol. 70, no. 4, pp. 3926-3931, Apr. 2021.

[206] M. Katwe, P. Ghare, and P. K. Sharma, "Robust NLOS bias mitigation for hybrid RSS-TOA based source localization under unknown transmission parameters," IEEE Wireless Commun. Lett., vol. 10, no. 3, pp. 542-546, Mar. 2021.

[207] X. Yang and F. Zhao, "Echo state network and echo state Gaussian process for non-line-of-sight target tracking," IEEE Syst. J., vol. 14, no. 3, pp. 3885-3892, Sep. 2020.

[208] C. He, Y. Yuan, and B. Tan, "Alternating direction method of multipliers for TOA-based positioning under mixed sparse LOS/NLOS environments," IEEE Access, vol. 9, pp. 28 407-28 412, Feb. 2021.

[209] K. Yu, K. Wen, Y. Li, S. Zhang, and K. Zhang, "A novel NLOS mitigation algorithm for UWB localization in harsh indoor environments," IEEE Trans. Veh. Technol., vol. 68, no. 1, pp. 686-699, Jan. 2019.

[210] B. Silva and G. P. Hancke, "Ranging error mitigation for through-the-wall non-line-of-sight conditions," IEEE Trans. Ind. Informat., vol. 16, no. 11, pp. 6903-6911, Nov. 2020.

[211] S. Angarano, V. Mazzia, F. Salvetti, G. Fantin, and M. Chiaberge, "Robust ultra-wideband range error mitigation with deep learning at the edge," Eng. Appl. Artificial Intell., vol. 102, pp. 1-9, June 2021.

[212] S. Sabour, N. Frosst, and G. E. Hinton, "Dy- 
namic routing between capsules," arXiv preprint arXiv:1710.09829, Nov. 2017.

[213] N. Saeed, H. Nam, T. Y. Al-Naffouri, and M.-S. Alouini, "A state-of-the-art survey on multidimensional scalingbased localization techniques," IEEE Commun. Surveys, Tuts., vol. 21, no. 4, pp. 3565-3583, 4th Quart. 2019.

[214] O. Jikun, "Quasi accurate detection of gross errors (QUAD)," Acta Geodaetica et Cartographic Sinica, vol. 28, no. 1, pp. 15-20, 1999.

[215] V. Barral, C. J. Escudero, J. A. García-Naya, and R. Maneiro-Catoira, "NLOS identification and mitigation using low-cost UWB devices," Sensors, vol. 19, no. 16, pp. 1-15, Aug. 2019.

[216] C. Lian Sang, B. Steinhagen, J. D. Homburg, M. Adams, M. Hesse, and U. Rückert, "Identification of NLOS and multi-path conditions in UWB localization using machine learning methods," Appl. Sci., vol. 10, no. 11, pp. 1-25, June 2020.

[217] X. Yang, J. Wang, D. Song, B. Feng, and H. Ye, "A novel NLOS error compensation method based IMU for UWB indoor positioning system," IEEE Sensors J., vol. 21, no. 9, pp. 11203-11212, May 2021.

[218] R. Zhang, Z. Zhao, X. Cheng, and L. Yang, "Overlapping coalition formation game based opportunistic cooperative localization scheme for wireless networks," IEEE Trans. Commun., vol. 65, no. 8, pp. 3629-3642, Aug. 2017.

[219] O. Hayat, R. Ngah, Z. Kaleem, S. Z. M. Hashim, and J. J. Rodrigues, "A survey on security and privacy challenges in device discovery for next-generation systems," IEEE Access, vol. 8, pp. 84 584-84 603, May 2020.

[220] C. E. O'Lone, H. S. Dhillon, and R. M. Buehrer, "A statistical characterization of localization performance in wireless networks," IEEE Trans. Wireless Commun., vol. 17, no. 9, pp. 5841-5856, Sep. 2018.

[221] D. S. Deif and Y. Gadallah, "An ant colony optimization approach for the deployment of reliable wireless sensor networks," IEEE Access, vol. 5, pp. 10 744-10 756, June 2017.

[222] B. Cao, X. Kang, J. Zhao, P. Yang, Z. Lv, and X. Liu, "Differential evolution-based 3-D directional wireless sensor network deployment optimization," IEEE Internet Things J., vol. 5, no. 5, pp. 3594-3605, Oct. 2018.

[223] H. Wymeersch, N. Garcia, H. Kim, G. Seco-Granados, S. Kim, F. Wen, and M. Fröhle, "5G mm wave downlink vehicular positioning," in Proc. IEEE Global Commun. Conf., Abu Dhabi, United Arab Emirates, pp. 206-212, Dec. 2018.

[224] B. Wang, Q. Xu, C. Chen, F. Zhang, and K. R. Liu, "The promise of radio analytics: A future paradigm of wireless positioning, tracking, and sensing," IEEE Signal Process. Mag., vol. 35, no. 3, pp. 59-80, May. 2018.

[225] J. Chen, W. Dai, Y. Shen, V. K. Lau, and M. Z. Win, "Power management for cooperative localization: A game theoretical approach," IEEE Trans. Signal Process., vol. 64, no. 24, pp. 6517-6532, Dec. 2016.

[226] D. Qiao and G. K. H. Pang, "A modified differential evolution with heuristic algorithm for nonconvex optimization on sensor network localization," IEEE Trans. Veh. Technol., vol. 65, no. 3, pp. 1676-1689, Mar. 2016.

[227] S. D. Correia, M. Beko, S. Tomic, and L. A. D. S. Cruz, "Energy-based acoustic localization by improved elephant herding optimization," IEEE Access, vol. 8, pp. 28 548-28 559, Feb.2020.

[228] L. A. Caceres-Najarro, I. Song, S. Tomic, and K. Kim, "Fast localization with unknown transmit power and path-loss exponent in WSNs based on RSS measurements," IEEE Commun. Lett., vol. 24, no. 12, pp. 27562760, Dec. 2020.

[229] R. Hummel, "Nestcout threat intelligence report: DDoS in a time of pandemic," Netscout Systems Inc., Westford, MA, USA, Rep. 2H2020, vol. 6, 2020.

[230] P. Zhang, S. G. Nagarajan, and I. Nevat, "Secure location of things (SLOT): Mitigating localization spoofing attacks in the internet of things," IEEE Internet Things J., vol. 4, no. 6, pp. 2199-2206, Dec. 2017.

[231] T. Zhang, X. Li, and Q. Zhang, "Location privacy protection: A power allocation approach," IEEE Trans. Commun., vol. 67, no. 1, pp. 748-761, Jan. 2019.

[232] Y. Li, S. Ma, G. Yang, and K. K. Wong, "Secure localization and velocity estimation in mobile IoT networks with malicious attacks," IEEE Internet Things J., vol. 8, no. 8, pp. 6878-6892, Apr. 2020.

[233] M. Beko and S. Tomic, "Towards secure localization in randomly deployed wireless networks," IEEE Internet Things J., Early Access 2021.

[234] J. Zhao, Y. Zhang, S. Ni, and Q. Li, "Bayesian cooperative localization with NLOS and malicious vehicle detection in GNSS-challenged environments," IEEE Access, vol. 8, pp. 85686-85697, May 2020.

[235] Q. Kong, R. Lu, F. Yin, and S. Cui, "Blockchain-based privacy-preserving driver monitoring for MaaS in the vehicular IoT," IEEE Trans. Veh. Technol., vol. 70, no. 4, pp. 3788-3799, Apr. 2021.

[236] D. Hendrycks, M. Mazeika, S. Kadavath, and D. Song, "Using self-supervised learning can improve model robustness and uncertainty," arXiv preprint arXiv:1906.12340, Oct. 2019. 